Research Article

\title{
Eigenvalue Spectrum and Synchronizability of Two Types of Double-Layer Star-Ring Networks with Hybrid Directional Coupling
}

\author{
Feimei Yang $\mathbb{D},{ }^{1}$ Zhen Jia $\mathbb{D},{ }^{1}$ and Yang Deng ${ }^{2}$ \\ ${ }^{1}$ College of Science, Guilin University of Technology, Guilin 541004, China \\ ${ }^{2}$ College of Science, Harbin Institute of Technology, Shenzhen 18213, China \\ Correspondence should be addressed to Zhen Jia; jjjzzz0@163.com
}

Received 19 December 2020; Revised 12 May 2021; Accepted 30 May 2021; Published 7 June 2021

Academic Editor: Francisco R. Villatoro

Copyright (c) 2021 Feimei Yang et al. This is an open access article distributed under the Creative Commons Attribution License, which permits unrestricted use, distribution, and reproduction in any medium, provided the original work is properly cited.

In this study, we studied the eigenvalue spectrum and synchronizability of two types of double-layer hybrid directionally coupled star-ring networks, namely, the double-layer star-ring networks with the leaf node pointing to the hub node (Network I) and the double-layer star-ring networks with the hub node pointing to the leaf node (Network II). We strictly derived the eigenvalue spectrum of the supra-Laplacian matrix of these two types of networks and analyzed the relationship between the synchronizability and the structural parameters of networks based on the master stability function theory. Furthermore, the correctness of the theoretical results was verified through numerical simulations, and the optimum structural parameters were obtained to achieve the optimal synchronizability.

\section{Introduction}

Complex networks are common in daily life, such as social networks [1], urban transportation networks, and virus spread networks. As the study of complex networks deepens, scholars realized that many networks, both in nature and in society, are highly complex and interdependent or interrelation; for example, in the transportation system, road networks, railway networks, aviation networks, and waterway transport networks are relatively independent and interrelated, which together constitute the superlarge network of the complex transportation system. The congestion of a subway may be related to the simultaneous arrival of trains, buses, and other buses and the instantaneous flow of passengers into the subway station. For another example, in human social activities, a network is formed with each individual as the hub, and each individual interacts with each other online or offline to form a complex social network. Therefore, the research on complex networks cannot be limited to simple regular or random single-layer unidirectional networks. In reality, mostly the networks are small- world, scale-free, or even more complex multilayer or directional coupling networks. At present, abundant research results have been achieved in the study of single-layer complex networks [2-9], while multilayer directional networks have become a new frontier research direction of complex networks.

Synchronization is a common set phenomenon in nature, such as the chorus of frogs and the simultaneous twinkling of fireflies. At the same time, people use synchronization to solve many problems in medical, physical, economic, and other fields. In recent years, synchronization and its control of chaos have been deeply studied by scholars [10-14]. Although the research on the synchronization of single-layer complex networks has been relatively mature [15-24], some theories and methods of single-layer networks are difficult to be directly applied to multilayer networks, and multilayer networks have richer and more complex dynamics than single-layer networks. Therefore, the synchronization of multilayer networks is a challenging topic.

At present, some preliminary results have been achieved in the research of multilayer complex networks 
synchronizability [25-28]. In [29], Gómez et al. studied the time scale of diffusion process on multiple networks in 2013 proposed the dynamic model of multilayer networks, and constructed the supra-Laplacian matrix, which provided an idea for later scholars to study multiple complex networks. In [28], $\mathrm{Xu}$ et al. studied the eigenvalue spectrum and synchronization of two-layer undirected star networks and compared its conclusion with the numerical simulation results of two-layer BA scale-free networks; it was found that the conclusions on the synchronization ability of these two kinds of networks are very similar. In [30], Sun et al. in 2017, respectively, expanded the star networks from leaf nodes to hub nodes and from hub nodes to leaf nodes from threelayer star networks to multilayer star networks, analyzed the eigenvalue spectrum and synchronization of unidirectional coupled star networks, and gave the analytic expression of eigenvalue. In [31], Deng et al. in 2019 discussed the eigenvalue spectrum and synchronization of multiplex chain networks. The star-ring network is a regular network derived from the star network. In [32], Deng et al. researched the relationship between the eigenvalue spectrum, structure parameters, and synchronizability of multilayer star-ring networks with no weight and no direction, especially analyzing the change of synchronizability under different interlayer coupling strengths. However, in our real complex network, whether it is a single-layer complex network or a multilayer complex network, edges between nodes have directions or weights. It is of more practical significance to investigate the synchronization of multilayer directed or weighted networks. If a double-layer star-ring network is combined with hybrid directional coupling, how does the supra-Laplacian matrix and the synchronizability change? Generally, for the eigenvalues of the multilayer hybrid directional coupling network, it is difficult to strictly derive the theoretical solution, mostly directly through the numerical simulation to explore the synchronization, and little previous research was conducted on the multilayer hybrid directionally coupling network synchronizability; therefore, a better understanding of the two layers of hybrid directionally coupling network to the topology of the synchronizability is needed. In this article, we studied the double-layer hybrid directionally coupling star-ring networks from leaf nodes to hub nodes and from hub nodes to leaf nodes, respectively. According to the network of Laplacian, its eigenvalue is strictly deduced analytically; then, the stability function method is reused to analyze the network structure parameters and their relationship with the network synchronizability. Finally, the correctness of the theoretical analysis was verified by the numerical simulation.

The rest of this article is arranged as follows. Section 2 explains some preliminaries. In Section 3, the eigenvalue spectrum and synchronizability of double-layer hybrid directionally coupled star-ring Network I are studied. Section 4 studies the eigenvalue spectrum and synchronizability of double-layer hybrid directionally coupled star-ring Network II. Finally, this article is concluded in Section 5 .

\section{Preliminaries}

2.1. Dynamics Model of Multilayer Networks. In an M-layer network, if there are $N$ nodes in each layer of the subnetwork, then the dynamic equation of the $i$ th node can be written as follows $[28,31-34]$ :

$$
\dot{x}_{i}^{K}=f\left(x_{i}^{K}\right)+a^{K} \sum_{j=1}^{N} \omega_{i j}^{K} H\left(x_{j}^{K}\right)+\sum_{L=1}^{M} d_{i}^{K L} \Gamma\left(x_{i}^{L}\right),
$$

where $1 \leq i \leq N, 1 \leq K \leq M ; x_{i}^{K} \in R^{n}$ is the state variable of the $i$ th node of the Kth layer. $f(\cdot): R^{n} \longrightarrow R^{n}$ is the dynamic equation of a single node; $a^{K}$ is the intralayer coupling strength of the Kth layer. Here, $\mathbf{W}^{K}=\left(\omega_{i j}^{K}\right) \in R^{N \times N}$ is the inner coupling matrix of the Kth layer. If there is an edge between the $i$ th node and the $j$ th node $(j \neq i)$ in the Kth layer, $\omega_{i j}^{K}=1$; otherwise, $\omega_{i j}^{K}=0$; there is

$$
\omega_{i i}^{K}=-\sum_{j=1, j \neq i}^{N} \omega_{i j}^{K}, \quad i, j=1,2, \ldots, N, K=1,2, \ldots, M .
$$

Then, $\mathbf{L}^{K}=-a^{K} \mathbf{W}^{K}$ is the Laplacian matrix of the $K$ th layer. Here, $H(\cdot): R^{n} \longrightarrow R^{n}$ is the intralayer coupling function. $d_{i}^{K L}$ is the interlayer coupling strength of the $i$ th node between the Kth layer and the Lth layer. Accordingly, if there is an edge between the $i$ th node of the Kth layer and the $i$ th node of the $L$ th layer, $d_{i}^{K L}=1$; otherwise, $d_{i}^{K L}=0$, so there is

$$
d_{i}^{K K}=-\sum_{L=1, L \neq K}^{M} d_{i}^{K L}, \quad L, K=1,2, \ldots, M .
$$

Then, $\mathbf{D}=\left(d_{i}^{K L}\right) \in R^{M \times M}$ is the interlayer negative Laplacian matrix between the $k$ th layer and the $L$ th layer. $\Gamma(\cdot): R^{n} \longrightarrow R^{n}$ is the interlayer coupling function.

Let $\mathscr{L}^{L}$ be the supra-Laplacian matrix of the intralayer and $\mathscr{L}^{I}$ be the supra-Laplacian matrix of the interlayer; then, the supra-Laplacian matrix $\mathscr{L}$ of model (1) can be written as

$$
\mathscr{L}=\mathscr{L}^{L}+\mathscr{L}^{I}
$$

where $\mathscr{L}^{L}$ represents the direct sum of the intralayer Laplacian matrix and $\mathscr{L}^{I}$ represents the Kronecker product between the interlayer Laplacian matrix and the $N \times N$ identity matrix, so there is

$$
\begin{aligned}
& \mathscr{L}^{L}=\left(\begin{array}{cccc}
L^{(1)} & 0 & \cdots & 0 \\
0 & L^{(2)} & \cdots & 0 \\
\vdots & \vdots & \ddots & \vdots \\
0 & 0 & \cdots & L^{(M)}
\end{array}\right)=\underset{K=1}{M} L^{(K)}, \quad K=1,2, \ldots, M, \\
& \mathscr{L}^{I}=L^{I \otimes I_{N \times N} .}
\end{aligned}
$$

In short, the supra-Laplacian matrix $\mathscr{L}$ of network model (1) is determined by the topology of the network, and the dynamic character will be influenced by many factors, 
such as the interlayer connections, the intralayer connections, and the different coupling directions and coupling strength between intralayers and interlayers. We focused on the effect of topology on synchronization dynamics in this article.

2.2. Judgment Method of Network Synchronizability. The synchronous region of model (1) represents the range of eigenvalues that achieve the network synchronizability. According to the master stability function theory (MSF) [35], there are four kinds of synchronous regions in a network generally.

(1) The synchronous region is unbounded $\left(\alpha_{1},+\infty\right)$, in which case the network synchronizability is determined by the minimum nonzero eigenvalue $\lambda_{2}$ of the corresponding supra-Laplacian matrix $\mathscr{L}$. The larger $\lambda_{2}$ is, the stronger the synchronizability is.

(2) The synchronous region is bounded $\left(\alpha_{1}, \alpha_{2}\right)$, and the network synchronizability is determined by the ratio $R$ between the maximum eigenvalue $\lambda_{\max }$ of the matrix and the minimum nonzero eigenvalue $\lambda_{2}$. The smaller $R=\left(\lambda_{\max } / \lambda_{2}\right)$ is, the stronger the synchronizability is.

(3) The synchronous region is the union of several unconnected regions. In this case, it is difficult to realize the synchronizability.

(4) When the synchronous region is an empty set, it cannot achieve complete synchronizability no matter how the topology and coupling strength change.

Since most of the synchronous regions are in the situation of (1) and (2), $\lambda_{2}$ and $R=\left(\lambda_{\max } / \lambda_{2}\right)$ are mainly used as indicators to investigate the network synchronizatbility when analyzing the synchronization.
In this article, we mainly study two types of double-layer star-ring networks, i.e., the double-layer star-ring network with hybrid directional coupling (Network I) from the leaf node to the hub node and the double-layer star-ring network with hybrid directional coupling (Network II) from hub nodes to leaf nodes. The synchronizability of these two networks, in the case that the synchronous region is unbounded and the synchronous region is bounded, is studied, respectively, and $\lambda_{2}$ and $R=\left(\lambda_{\max } / \lambda_{2}\right)$ are used as indexes of the synchronizability.

\section{The Eigenvalue Spectrum and Synchronizability of Double-Layer Hybrid Directionally Coupled Star-Ring Network I}

3.1. The Structure Model of Network I. In this article, the structure of Network I model researched is shown in Figure 1. It is assumed that the topological structure of each layer is identical, and the interlayer hub nodes and hub nodes, leaf nodes, and leaf nodes of subnetworks are in oneto-one corresponding coupling. Suppose that the connections of the intralayer nodes are directional; that is, leaf nodes point to hub nodes and the directions between leaf nodes and leaf nodes are consistent, while the connections of the interlayer nodes are undirectional. Let the intralayer coupling strength of leaf nodes be $a_{0}$, the intralayer coupling strength between leaf nodes and hub nodes be $a$, the interlayer coupling strength of leaf nodes be $d$, the interlayer coupling strength of hub nodes be $d_{0}$, and the number of nodes of subnetwork be $N$ (a total of two layers).

3.2. The Eigenvalue Spectrum and Synchronizability of Network I. First, according to the structure of the Network I model, its supra-Laplacian matrix $\mathscr{L}_{1}$ can be written as

$$
\mathscr{L}_{1}=\left[\begin{array}{cccccccccccc}
d_{0} & 0 & 0 & \cdots & 0 & 0 & -d_{0} & 0 & 0 & \cdots & 0 & 0 \\
-a & a+d+a_{0} & 0 & \cdots & 0 & -a_{0} & 0 & -d & 0 & \cdots & 0 & 0 \\
-a & -a_{0} & a+d+a_{0} & \cdots & 0 & 0 & 0 & 0 & -d & \cdots & 0 & 0 \\
\vdots & \vdots & \vdots & \ddots & \vdots & \vdots & \vdots & \vdots & \vdots & \ddots & \vdots & \vdots \\
-a & 0 & 0 & \cdots & a+d+a_{0} & 0 & 0 & 0 & 0 & \cdots & -d & 0 \\
-a & 0 & 0 & \cdots & -a_{0} & a+d+a_{0} & 0 & 0 & 0 & \cdots & 0 & -d \\
-d_{0} & 0 & 0 & \cdots & 0 & 0 & d_{0} & 0 & 0 & \cdots & 0 & 0 \\
0 & -d & 0 & \cdots & 0 & 0 & -a & a+d+a_{0} & 0 & \cdots & 0 & -a_{0} \\
0 & 0 & -d & \cdots & 0 & 0 & -a & -a_{0} & a+d+a_{0} & \cdots & 0 & 0 \\
\vdots & \vdots & \vdots & \ddots & \vdots & \vdots & \vdots & \vdots & \vdots & \ddots & \vdots & \vdots \\
0 & 0 & 0 & \cdots & -d & 0 & -a & 0 & 0 & \cdots & a+d+a_{0} & 0 \\
0 & 0 & 0 & \cdots & 0 & -d & -a & 0 & 0 & \cdots & -a_{0} & a+d+a_{0}
\end{array}\right]
$$




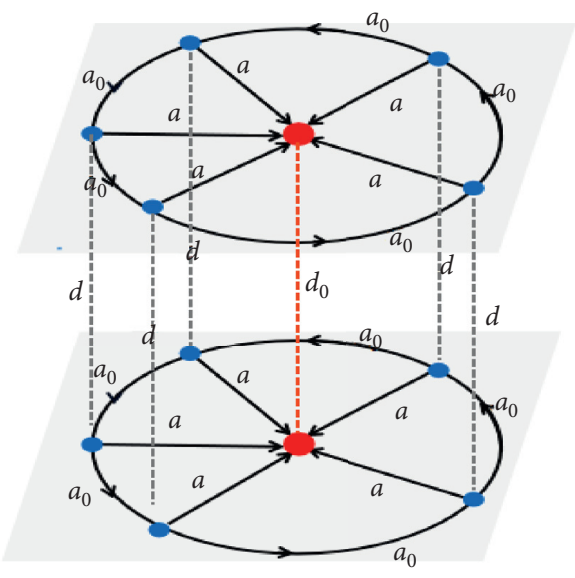

Figure 1: Schematic diagram of the structural model of Network I.

According to the determinant property $\left|\begin{array}{ll}A & B \\ B & A\end{array}\right|=\mid A+$ $B|| A-B \mid$ of the block matrix, the characteristic polynomial of the supra-Laplacian matrix $\mathscr{L}_{1}$ of Network I can be derived as follows:

$$
\begin{aligned}
& \left|\lambda I-\widetilde{\mathscr{L}}_{1}\right|=\left|\begin{array}{cccccc}
\lambda & 0 & 0 & \cdots & 0 & 0 \\
a & \lambda-a-a_{0} & 0 & \cdots & 0 & a_{0} \\
a & a_{0} & \lambda-a-a_{0} & \cdots & 0 & 0 \\
\vdots & \vdots & \vdots & \ddots & \vdots & \vdots \\
a & 0 & 0 & \cdots & \lambda-a-a_{0} & 0 \\
a & 0 & 0 & \cdots & a_{0} & \lambda-a-a_{0}
\end{array}\right|_{N \times N} \\
& \left|\begin{array}{cccccc}
\lambda-2 d_{0} & 0 & 0 & \cdots & 0 & 0 \\
a & \lambda-a-2 d-a_{0} & 0 & \cdots & 0 & a_{0} \\
a & a_{0} & \lambda-a-2 d-a_{0} & \cdots & 0 & 0 \\
\vdots & \vdots & \vdots & \ddots & \vdots & \vdots \\
a & 0 & 0 & \cdots & \lambda-a-2 d-a_{0} & 0 \\
a & 0 & 0 & \cdots & a_{0} & \lambda-a-2 d-a_{0}
\end{array}\right|_{N \times N} \\
& =\lambda\left[(-1)^{2+N} a_{0}{ }^{N-1}+\left(\lambda-a-a_{0}\right)^{N-1}\right]\left(\lambda-2 d_{0}\right)\left[(-1)^{2+N} a_{0}{ }^{N-1}+\left(\lambda-a-a_{0}-2 d\right)^{N-1}\right] \text {. }
\end{aligned}
$$

The eigenvalues of Network I are calculated as follows.

When the number of subnetwork nodes $N$ is odd,

$$
\lambda=0,2 d_{0}, \underbrace{a, \ldots, a}_{(N-1) / 2}, \underbrace{a+2 a_{0}, \ldots, a+2 a_{0}}_{(N-1) / 2}, \underbrace{a+2 d, \ldots, a+2 d}_{(N-1) / 2}, \underbrace{a+2 a_{0}+2 d, \ldots, a+2 a_{0}+2 d}_{(N-1) / 2} .
$$

When the number of subnetwork nodes $N$ is even,

$$
\lambda=0,2 d_{0}, \underbrace{a, \ldots, a}_{N-1}, \underbrace{a+2 d, \ldots, a+2 d}_{N-1} .
$$

According to the odd and even case of the number of subnetwork nodes $N$, there are also two cases when analyzing the Network I synchronizability.
(1) $N$ is odd, where $a+2 a_{0}, a+2 d, a+2 a_{0}+2 d$ andaare $((N-1) / 2)$ multiple roots. $\lambda_{\max }$ is the maximum eigenvalue of $\mathscr{L}_{1}$ and $\lambda_{2}$ is the minimum nonzero eigenvalue of $\mathscr{L}_{1}$; then, $\lambda_{2}=\min \left\{2 d_{0}, a\right\}$,

$$
R=\frac{\lambda_{\max }}{\lambda_{2}}=\frac{a+2 a_{0}+2 d}{\min \left\{2 d_{0}, a\right\}}
$$


For the convenience of doing analysis, the relationship between $\lambda_{2}, R=\left(\lambda_{\max } / \lambda_{2}\right)$ and structure parameters is summarized in Table 1.

According to the MSF theory, when the synchronous region is unbounded, the synchronizability of Network $\mathrm{I}$ is determined by $\lambda_{2}$. The larger $\lambda_{2}$ is, the stronger the synchronizability is. When the synchronous region is bounded, the synchronizability of Network I is determined by $R=\left(\left(a+2 a_{0}+2 d\right) / 2 d_{0}\right)$. The smaller $R$ is, the stronger the synchronizability is. From the expressions of $\lambda_{2}$ and $R$, it can be seen that whether the synchronous region of Network I is unbounded or bounded, the synchronizability is independent of the number of nodes $N$ of the subnetwork in combination with the changes of $\lambda_{2}$ and $R$ with the structure parameters in Table 1 . Therefore, the relationship between the synchronizability of Network I and the structure parameters is shown in Table 2.

As can be seen from Table 2 , in the case that $N$ is odd, when the synchronous region is unbounded, the synchronizability of Network I is determined by $a, d_{0}$, and the synchronizability becomes stronger when $a, d_{0}$ are enlarged. When the synchronous region is bounded, the synchronizability of Network I is related to $a, a_{0}, d$ and $d_{0}$ but not to the number of subnetwork nodes. When the intralayer coupling strengths from leaf nodes to hub nodes are large, the increase of $d_{0}$ enhances the synchronizability, whereas the synchronizability will be weakened by increasing $a, a_{0}, d$. When the intralayer coupling strengths from leaf nodes to hub nodes are small, only the increase of $a$ can strengthen the synchronizability; increasing $a_{0}, d$ will weaken the synchronizability.

(2) $N$ is even. $\lambda_{2}=\min \left\{2 d_{0}, a\right\}, \lambda_{\max }=a+2 d$, $R=\left(\lambda_{\max } / \lambda_{2}\right)=\left((a+2 d) / \min \left\{2 d_{0}, a\right\}\right)$. Then, the relationship between $\lambda_{2}, R=\left(\lambda_{\max } / \lambda_{2}\right)$ and the structural parameters are shown in Table 3.

According to the MSF theory, when the synchronous region is unbounded, the synchronizability of Network I is determined by $\lambda_{2}$. When the synchronous region is bounded, the synchronizability is determined by $R$. Therefore, the relationships between the synchronizability and $a, d, d_{0}$ are obtained as shown in Table 4.

As is shown in Table 4, when the synchronous region is unbounded, the synchronizability is only related to $a, d_{0}$, which indicates that the strong coupling strength between the interlayer coupling strength and the intralayer coupling strength cannot determine the synchronizability. When the synchronous region is bounded, the synchronizability is related to $a, d, d_{0}$. When the interlayer coupling strength between the hub nodes is small, increasing $a, d$ will weaken the synchronizability of the Network I, but the synchronizability with the increase of $d_{0}$ will strengthen. When the intralayer coupling strength between the leaf nodes is small, increasing $a$ will enhance the synchronizability, while increasing $d$ will weaken the synchronizability.

3.3. Numerical Simulation of Network I Synchronizability. In this section, we verify the correctness of the above theoretical results through a large number of numerical simulations and further explore the value of the structural parameters that enable Network I to achieve optimal synchronizability. Design parameters within the limited scope of the theoretical results after many times of experimental simulation were obtained and a group of more representative data was selected, and as long as the design parameters are within the limited range, the value of the control performance is the same. Furthermore, all the numerical simulations in this article directly use the functions in MATLAB software to calculate the supra-Laplacian eigenvalues of the network, which can ensure the efficiency and stability of the algorithm, which is easy to implement because it does not require high time and space complexity.

According to the study in the previous sections, for Network I, when the number of subnetwork nodes is odd, the synchronizability is related to the intralayer coupling strength from leaf nodes to hub nodes, the intralayer coupling strength between the leaf nodes, the interlayer coupling strength between the hub nodes, and the interlayer coupling strength between the leaf nodes. When the number of subnetwork nodes is even, the synchronizability is associated with the intralayer coupling strength from the leaf nodes to the hub nodes, the interlayer coupling strength between the leaf nodes, and the interlayer coupling strength between the hub nodes. Whether the number of subnetwork nodes is odd or even, the number of subnetwork nodes does not affect the synchronizability. In the following numerical simulations, we will take $N=199$ (odd) and $N=200$ (even) for numerical experiments to verify the above conclusions. All the numerical simulations in this article directly use the functions in MATLAB software to calculate the supraLaplacian eigenvalues of the network, which can ensure the efficiency and stability of the algorithm.

(1) $N$ is odd. In the numerical simulations, we take $N=199, a_{0}=0.5, d=1$, and $d_{0}=0.1$ to investigate the change of network synchronizability with the intralayer coupling strength from the leaf nodes to the hub nodes. The simulation results are shown in Figure 2. For the case of the unbounded synchronous region (Figure 2(a)), when $a<2 d_{0}, \lambda_{2}$ increases with the increase of $a$, so the synchronizability strengthens with the increase of $a$. When $a$ reaches a threshold value $2 d_{0}$, the synchronizability remains unchanged. Then, $2 d_{0}$ is the optimal parameter when the synchronizability reaches the maximum. For the case of bounded synchronous region (Figure 2(b)), when $a<2 d_{0}$, $R$ becomes less with the increase of $a$. When $a$ is equal to the threshold value $2 d_{0}, R$ decreases to the minimum; that is, $2 d_{0}$ is the optimal structural parameter to make the synchronization reach optimal. When $a>2 d_{0}, R$ increases slowly with 
TABLE 1: The change of $\lambda_{2}$ and $R$ with the increase of $a, a_{0}, d$, and $d_{0}$ of Network I when $N$ is odd.

\begin{tabular}{llcccc}
\hline & & Increase of $a$ & Increase of $a_{0}$ & Increase of $d$ & Increase of $d_{0}$ \\
\hline$\lambda_{2}=\min \left\{2 d_{0}, a\right\}$ & $a>2 d_{0}$ & Unchanged & Unchanged & Unchanged & Increased \\
& $a<2 d_{0}$ & Increased & Unchanged & Unchanged & Unchanged \\
& $a>2 d_{0}$ & Increased & Increased & Increased & Decreased \\
& $a<2 d_{0}$ & Decreased & Increased & Increased & Unchanged \\
\hline
\end{tabular}

TABle 2: The change of synchronizability with the increase of $a, a_{0}, d$, and $d_{0}$ of Network I when $N$ is odd.

\begin{tabular}{lccccc}
\hline & & Increase of $a$ & Increase of $a_{0}$ & Increase of $d$ & Increase of $d_{0}$ \\
\hline \multirow{2}{*}{ The unbounded synchronous region } & $\lambda_{2}=2 d_{0}$ & Unchanged & Unchanged & Unchanged & Strengthened \\
& $\lambda_{2}=a$ & Strengthened & Unchanged & Unchanged & Unchanged \\
\multirow{2}{*}{ The bounded synchronous region } & $R=\left(\left(a+2 a_{0}+2 d\right) / 2 d_{0}\right)$ & Weakened & Weakened & Weakened & Strengthened \\
& $R=\left(\left(a+2 a_{0}+2 d\right) / a\right)$ & Strengthened & Weakened & Weakened & Unchanged \\
\hline
\end{tabular}

TABLE 3: The change of $\lambda_{2}$ and $R$ with the increase of $a, d$, and $d_{0}$ of Network I when $N$ is even.

\begin{tabular}{lcccc}
\hline & & Increase of $a$ & Increase of $d$ & Increase of $d_{0}$ \\
\hline$\lambda_{2}=\min \left\{2 d_{0}, a\right\}$ & $a>2 d_{0}$ & Unchanged & Unchanged & Increased \\
\multirow{2}{*}{$R=\left((a+2 d) / \min \left\{2 d_{0}, a\right\}\right)$} & $a<2 d_{0}$ & Increased & Unchanged & Unchanged \\
& $a>2 d_{0}$ & Increased & Increased & Decreased \\
\hline
\end{tabular}

TABle 4: The change of synchronizability with the increase of $a, d$, and $d_{0}$ of Network I when $N$ is even.

\begin{tabular}{|c|c|c|c|c|}
\hline & & Increase of $a$ & Increase of $d$ & Increase of $d_{0}$ \\
\hline \multirow{2}{*}{ The unbounded synchronous region } & $\lambda_{2}=2 d_{0}$ & Unchanged & Unchanged & Strengthened \\
\hline & $\lambda_{2}=a$ & Strengthened & Unchanged & Unchanged \\
\hline \multirow{2}{*}{ The bounded synchronous region } & $R=\left((a+2 d) / 2 d_{0}\right)$ & Weakened & Weakened & Strengthened \\
\hline & $R=((a+2 d) / a)$ & Strengthened & Weakened & Unchanged \\
\hline
\end{tabular}

the increase of $a$. In other words, the synchronizability strengthens at first and then weakens slowly. Take $N=199, a=1, d=1$, and $d_{0}=0.1$ to investigate how the synchronizability changes with the intralayer coupling strength $a_{0}$ between the leaf nodes for $a>2 d_{0}$, and take $N=199, a=0.1, d=1$, and $d_{0}=0.1$ to investigate how the synchronizability changes with the intralayer coupling strength $a_{0}$ of the leaf nodes for $a<2 d_{0}$, as shown in Figure 3. For the case that the synchronous region is unbounded (Figure 3(a)), when $a>2 d_{0}, \lambda_{2}$ is unchanged no matter how $a_{0}$ changes. When $a<2 d_{0}, \lambda_{2}$ remains unchanged with the increase of $a_{0}$, indicating that the synchronizability remains unchanged. For the case where the synchronous region is bounded (Figure 3(b)), when $a>2 d_{0}, R$ increases rapidly with the increase of $a_{0}$. When $a<2 d_{0}, R$ increases slowly with the increase of $a_{0}$, and the influence of $a$ on synchronizability is greater than that of $2 d_{0}$, but the synchronizability is generally weaker. It can be obtained that the smaller the intralayer coupling strength between the leaf nodes is, the better the synchronizability is.
As is shown in Figure 4, we take $N=199, a=1, a_{0}=$ $0.5, d_{0}=0.1$ to examine the change of synchronizability with the increase of the interlayer coupling strength $d$ (when $a>2 d_{0}$ ) between the leaf nodes, and take $N=199, a=0.1, a_{0}=0.5, d_{0}=0.1$ to investigate the change of synchronizability with the increase of the interlayer coupling strength $d$ (when $a<2 d_{0}$ ) of the leaf nodes. Figure 4(a) shows that when $a>2 d_{0}, \lambda_{2}$ is not influenced by the increase of $d$. When $a<2 d_{0}, \lambda_{2}$ remains unchanged with the increase of $d$. Thus, the synchronizability is not affected by $d$ for the unbounded synchronous region. Figure 4(b) displays that when $a>2 d_{0}, R$ increases slowly with the increase of $d$. When $a<2 d_{0}, R$ increases rapidly with the increase of $d$ for the bounded synchronization domain. The interlayer coupling strength from the leaf nodes to the hub nodes $a$ has a great influence on the synchronizability, while the synchronizability is weakened with the increase of $d$. Therefore, a smaller $d$ is favorable for Network I to achieve optimal synchronizability.

As shown in Figure 5, we take $N=199, a=1, a_{0}=$ 0.5 , and $d=1$ to investigate the change of network 


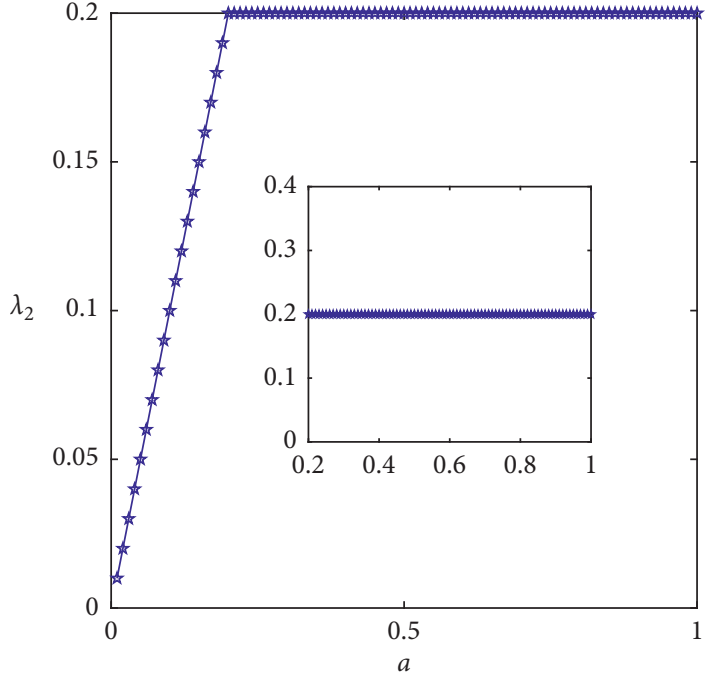

(a)

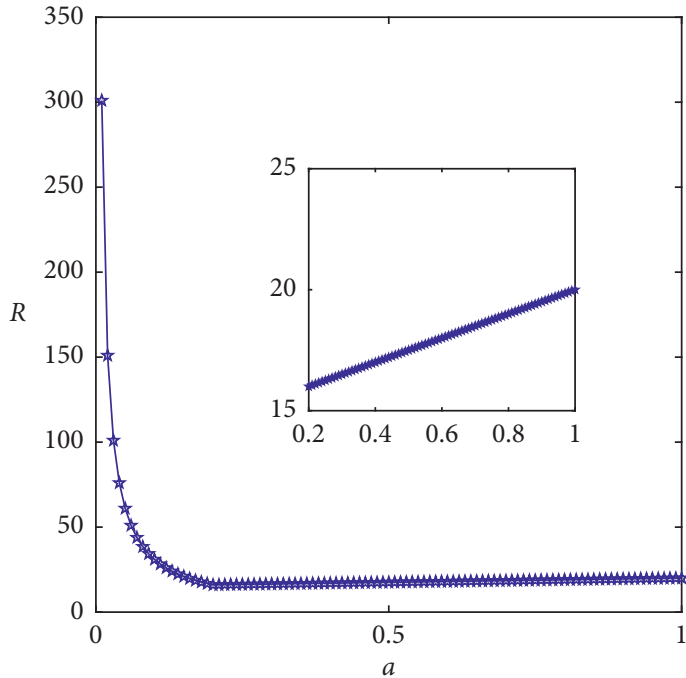

(b)

Figure 2: When $N$ is odd, the synchronizability of Network I changes with the intralayer coupling strength $a$ from the leaf nodes to the hub nodes. (a) $\lambda_{2}$ changes with $a$ ( $a$ changes from 0.01 to 1 ) (subgraph: $\lambda_{2}$ changes with $a$ (a changes from 0.2 to 1$)$ ); (b) $R$ changes with $a$ ( $a$ changes from 0.01 to 1 ) (subgraph: $R$ changes with $a$ ( $a$ changes from 0.2 to 1$)$ ).

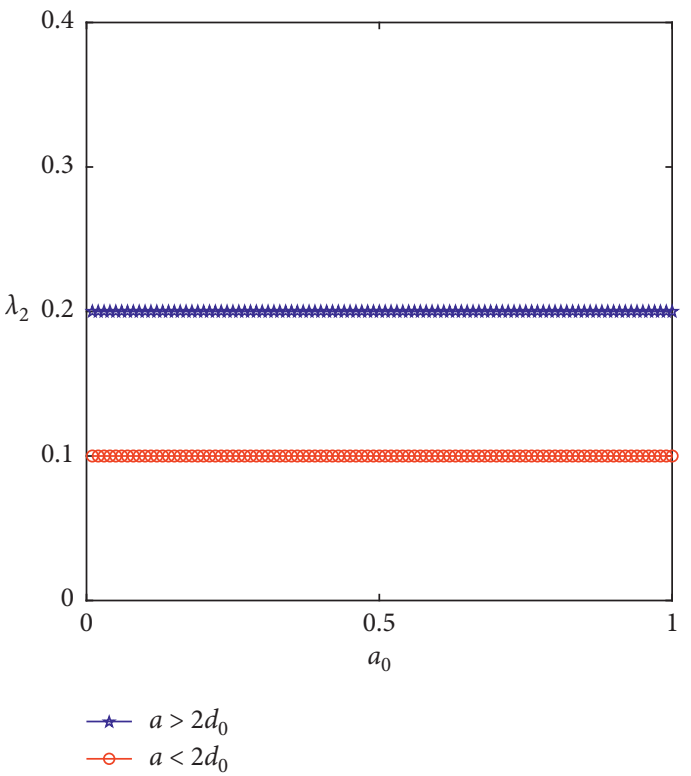

(a)

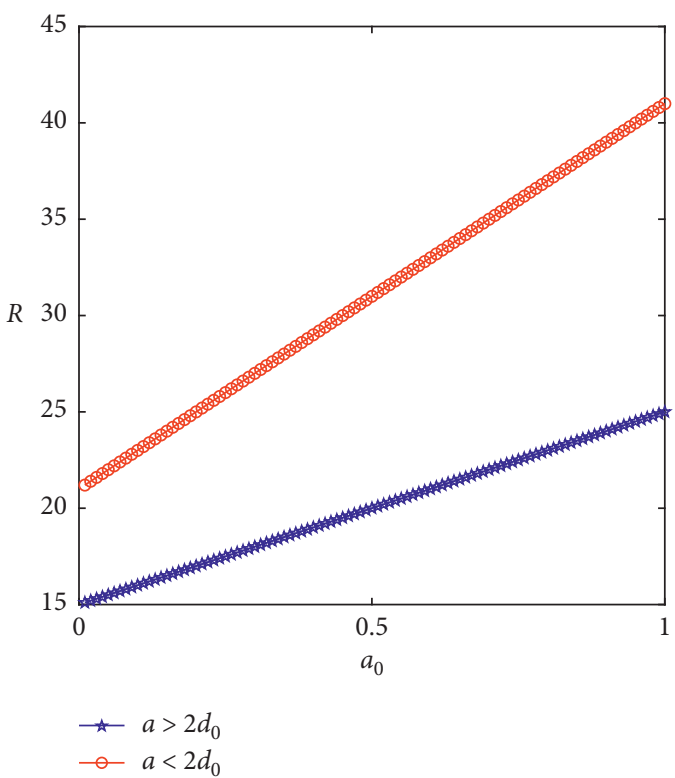

(b)

FIGURE 3: When $N$ is odd, the synchronizability of Network I changes with the intralayer coupling strength $a_{0}$ of the leaf nodes. (a) $\lambda_{2}$ changes with $a_{0}$ ( $a_{0}$ changes from 0.01 to 1 ); (b) $R$ changes with $a_{0}$ ( $a_{0}$ changes from 0.01 to 1 ).

synchronizability with the increase of the interlayer coupling strength $d_{0}$ between the hub nodes. In the case where the synchronous region is unbounded (Figure 5(a)), when $a>2 d_{0}, \lambda_{2}$ increases with the increase of $d_{0}$, and when $d_{0}$ reaches the threshold value $(a / 2), \lambda_{2}$ is not affected by $d_{0}$. In this case, $(a / 2)$ is the optimal structural parameter that reaches the optimal synchronizability; that is, the synchronizability strengthens at first and then remains unchanged with the increase of $d_{0}$. In the case where the synchronous region is bounded (Figure 5(b)), when $a>2 d_{0}, R$ decreases, with the increase of $d_{0}$, and when $d_{0}$ is about $(a / 2), R$ starts to remain unchanged; that is, $(a / 2)$ is the optimal structural parameter that makes the synchronizability reach the optimal. Thus, with the increase of $d_{0}$, the synchronizability at first strengthens and then remains unchanged. 


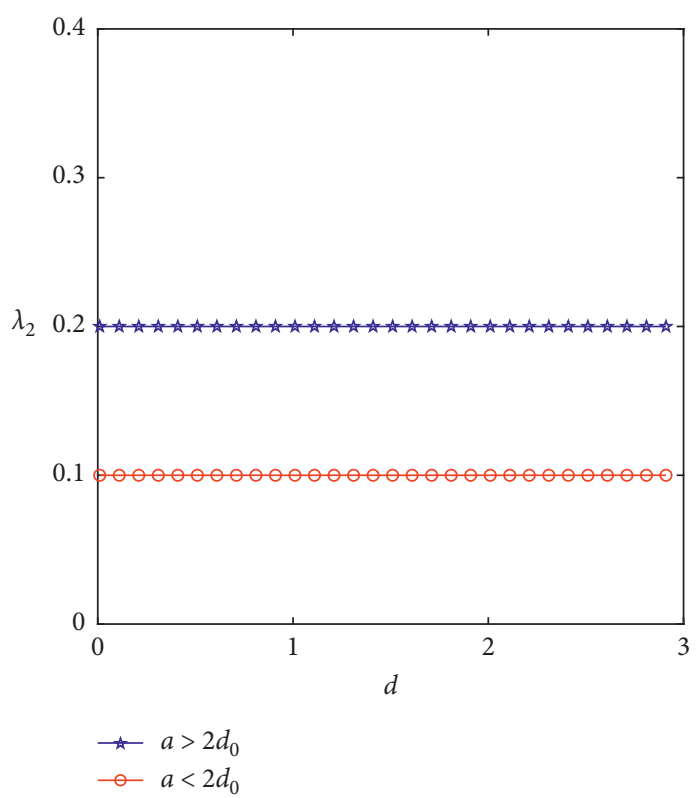

(a)

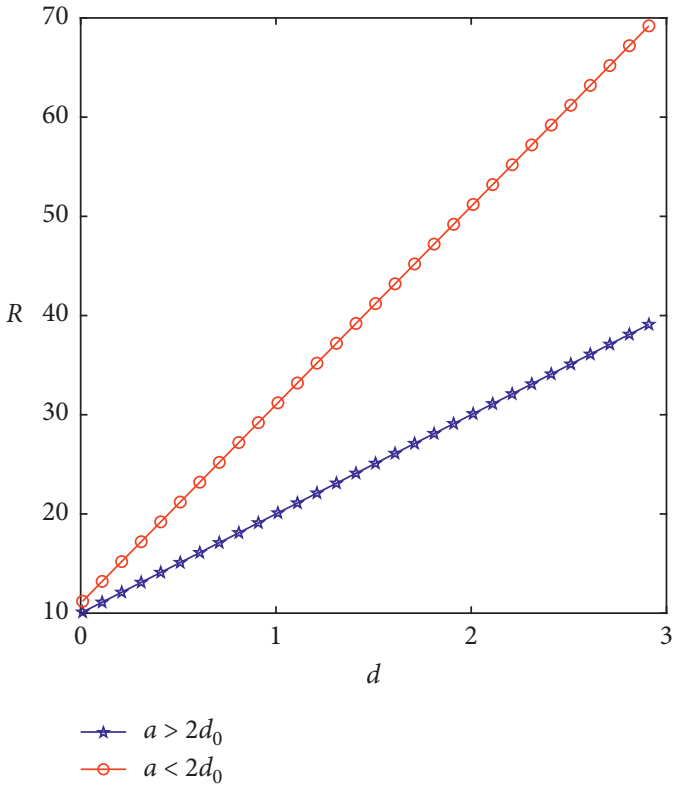

(b)

FIGURE 4: When $N$ is odd, the synchronizability of Network I changes with the interlayer coupling strength $d$ of the leaf nodes. (a) $\lambda_{2}$ changes with $d$ ( $d$ changes from 0.01 to 3 ); (b) $R$ changes with $d$ ( $d$ changes from 0.01 to 3 ).

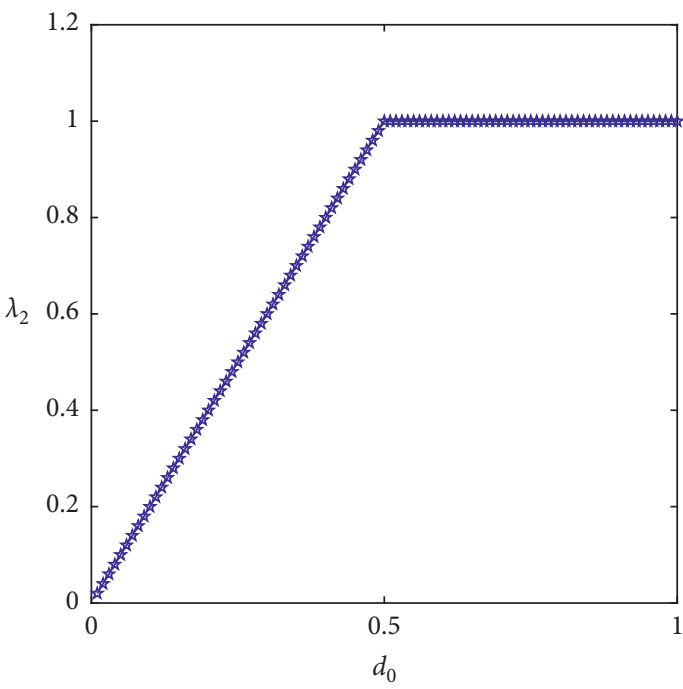

(a)

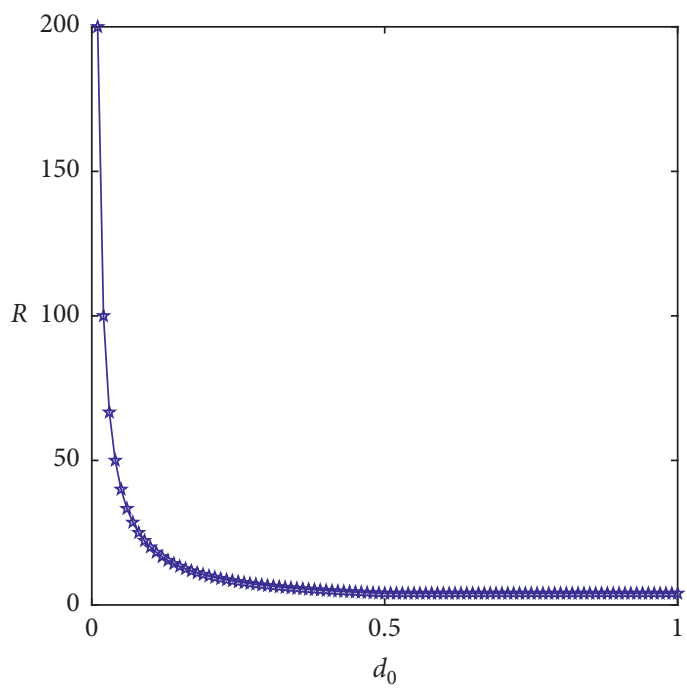

(b)

Figure 5: When $N$ is odd, the synchronizability of Network I changes with the interlayer coupling strength $d_{0}$ of the leaf nodes. (a) $\lambda_{2}$ changes with $d_{0}$ ( $d_{0}$ changes from 0.01 to 1 ); (b) $R$ changes with $d_{0}$ ( $d_{0}$ changes from 0.01 to 1 ).

(2) $N$ is even. As is shown in Figure 6, take $N=200, a_{0}=0.5, d=1, d_{0}=0.1$ to investigate how the synchronizability changes with the increase of the coupling strength $a$ from the leaf nodes to the hub nodes. In the unbounded synchronous region (Figure 6(a)), when $a<2 d_{0}, \lambda_{2}$ increases with the increase of $a$, so the synchronizability strengthens with the increase of $a$. When $a$ reaches the threshold value of $2 d_{0}$, the synchronizability is invariant, and then $2 d_{0}$ is the optimal structural parameter with the optimal synchronizability. In the bounded synchronous region (Figure 6(b)), when $a<2 d_{0}$, as $a$ increases, $R$ is increasingly small. When $a$ is equal to the threshold value $2 d_{0}, R$ decreases to the minimum. Therefore, $2 d_{0}$ is the optimal structural parameter that achieves the optimal network synchronizability. When $a>2 d_{0}, R$ slowly increases along with the augment of $a$, which 


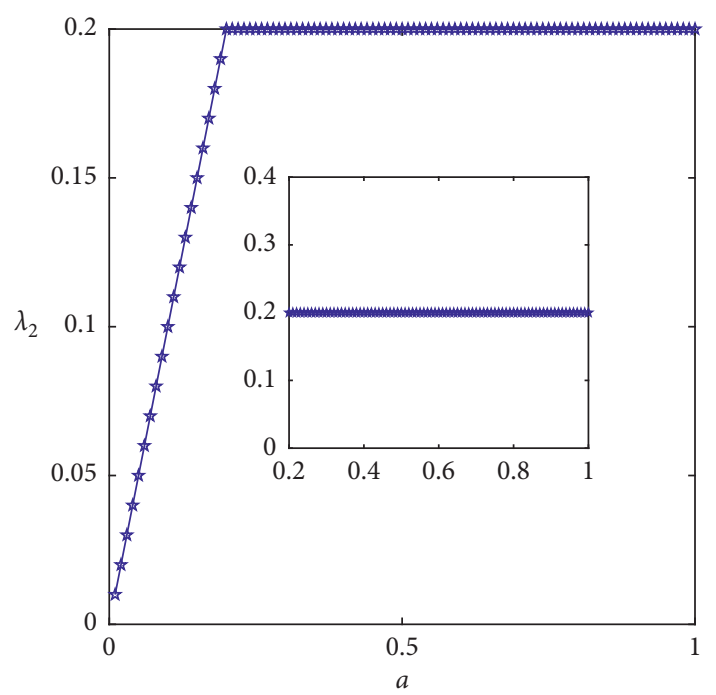

(a)

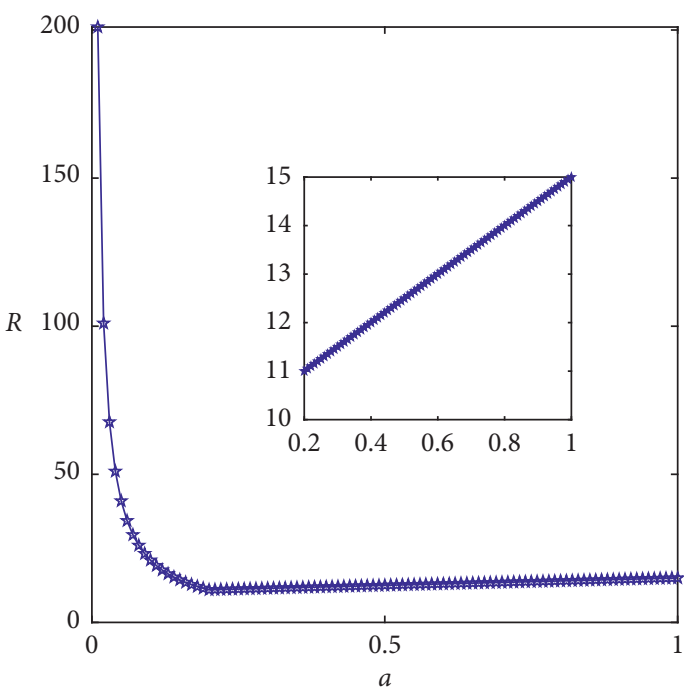

(b)

Figure 6: When $N$ is even, the synchronizability of Network I changes with the intralayer coupling strength $a$ from the leaf nodes to the hub nodes. (a) $\lambda_{2}$ changes with $a$ ( $a$ changes from 0.01 to 1 ) (subgraph: $\lambda_{2}$ changes with $a$ ( $a$ changes from 0.2 to 1$)$ ); (b) $R$ changes with $a$ ( $a$ changes from 0.01 to 1 ) (subgraph: $R$ changes with $a$ ( $a$ changes from 0.2 to 1$)$ ).

indicates that the synchronizability strengthens after the first slowly weaken.

We take $N=200, a=1, a_{0}=0.5, d_{0}=0.1$ to examine how the synchronizability changes with the increase of the interlayer coupling strength $d$ between the leaf nodes. Take $N=200, a=0.1, a_{0}=0.5, d_{0}=0.1$ to investigate how the network synchronizability changes with the increase of the interlayer coupling strength $d$ between the leaf nodes, as shown in Figure 7. Figure 7(a) shows that when $a>2 d_{0}, \lambda_{2}$ remains unchanged, in the increase of $d$. When $a<2 d_{0}, \lambda_{2}$ is not affected by $d$, indicating that the synchronizability remains unchanged, with the unbounded synchronous region. Notice from Figure 7(b) that when $a>2 d_{0}, R$ increases slowly with the increase of $d$; when $a<2 d_{0}, R$ increases rapidly with the increase of $d$, so the synchronizability weakens with the increase of $d$, with the bounded synchronous region.

Take $N=200, a=1, a_{0}=0.5, d=1$ to investigate how the synchronizability changes with the increase of the interlayer coupling strength $d_{0}$ between the hub nodes (Figure 8). When the synchronous region is unbounded (Figure 8(a)), when $a>2 d_{0}, \lambda_{2}$ increases with the increase of $d_{0}$, and when $d_{0}$ reaches the threshold value $(a / 2)$, the synchronizability remains unchanged; that is, $(a / 2)$ is the optimal structural parameter to achieve optimal synchronizability, and the synchronizability at first strengthens and then remains unchanged with the increase of $d_{0}$. In the case where the synchronous region is bounded (Figure $8(b)$ ), when $a>2 d_{0}, R$ decreases with the increase of $d_{0}$; when $d_{0}$ increases to $(a / 2)$, it has no influence on $R$. Therefore, $(a / 2)$ is the optimal structural parameter to reach the optimal synchronizability; that is, with the increase of $d_{0}$, the synchronizability at first strengthens and then remains unchanged.

\section{The Eigenvalue Spectrum and Synchronizability of Double-Layer Hybrid Directionally Coupled Star-Ring Network II}

4.1. The Structure Model of Network II. The structure model of Network II studied in this section is shown in Figure 9. It is assumed that the topological structure of each layer of the double-layer hybrid directional coupled star-ring network is identical, and the interlayer hub nodes and hub nodes, the leaf nodes, and the leaf nodes of the doublelayer subnetwork are in one-to-one coupling. Provided that the connections between the intralayer nodes are directional; that is, from the hub nodes to the leaf nodes, the intralayer direction between the leaf nodes and the leaf nodes is consistent, while the interlayer connection is undirected. Let the intralayer coupling strength between the leaf nodes be $a_{0}$, the intralayer coupling strength from the hub nodes to the leaf nodes be $a$, the interlayer coupling strength between the leaf nodes be $d$, the interlayer coupling strength between the hub nodes be $d_{0}$, and the number of nodes in each layer subnetwork be $N$ (a total of two layers of the Network II).

4.2. The Eigenvalue Spectrum and Synchronizability of Network II. According to the structural model of Network II, the supra-Laplacian matrix $\mathscr{L}_{2}$ of its network is as follows: 


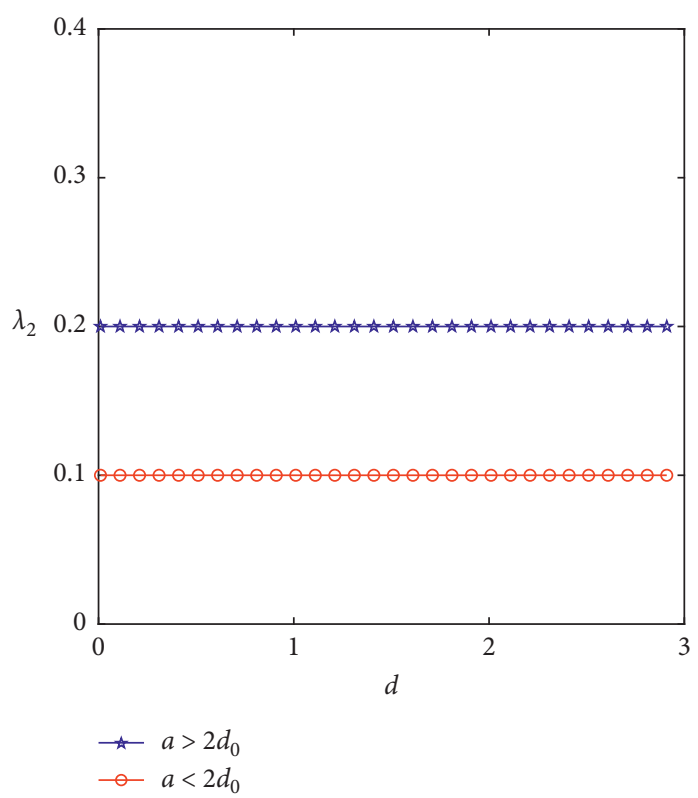

(a)

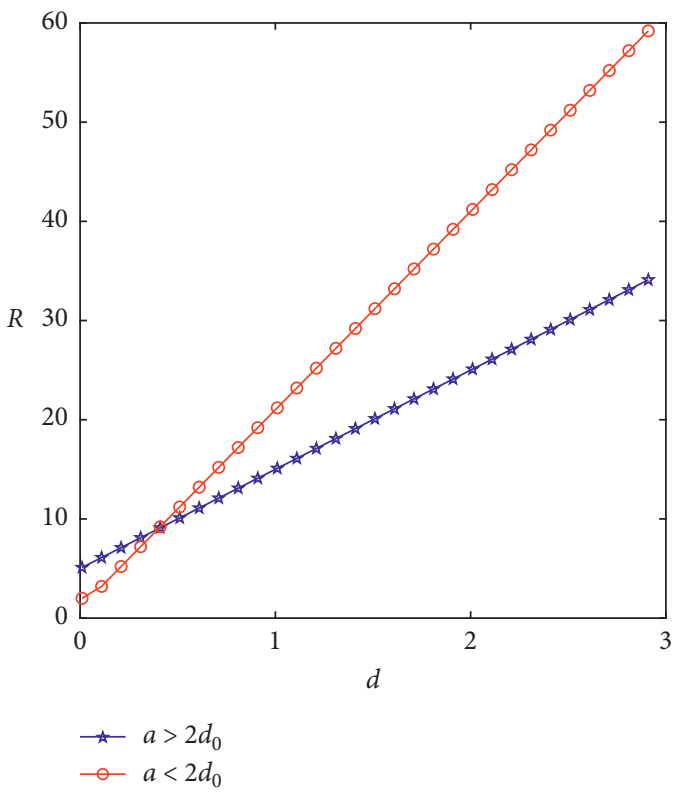

(b)

Figure 7: When $N$ is even, the synchronizability of Network I changes with the interlayer coupling strength $d$ of the leaf nodes. (a) $\lambda_{2}$ changes with $d$ ( $d$ changes from 0.01 to 3 ); (b) $R$ changes with $d$ ( $d$ changes from 0.01 to 3 ).

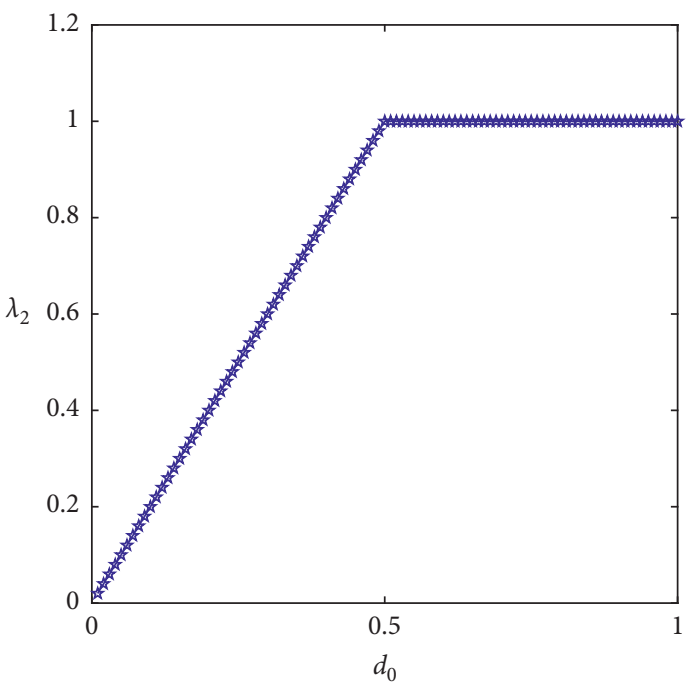

(a)

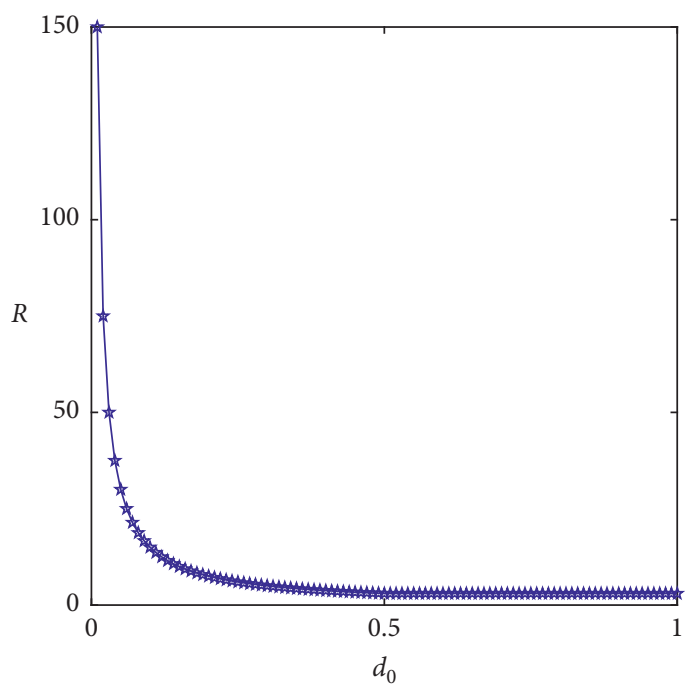

(b)

Figure 8: When $N$ is even, the synchronizability of Network I changes with the interlayer coupling strength $d_{0}$ of the leaf nodes. (a) $\lambda_{2}$ changes with $d_{0}$ ( $d_{0}$ changes from 0.01 to 1 ); (b) $R$ changes with $d_{0}$ ( $d_{0}$ changes from 0.01 to 1 ). 


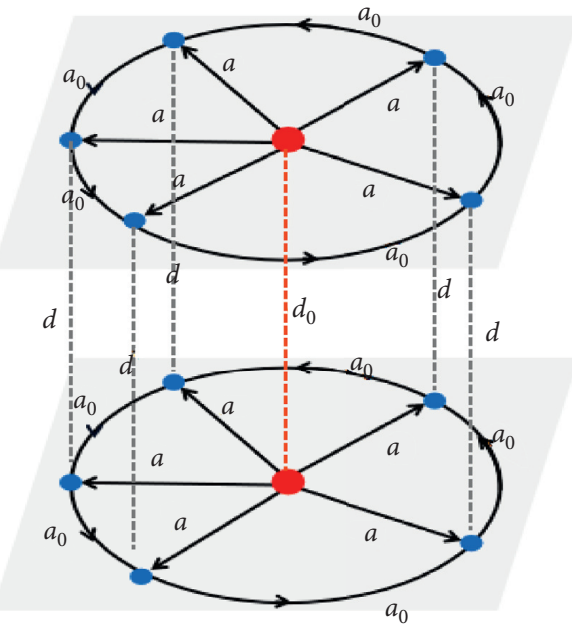

Figure 9: Schematic diagram of the structural model of Network II.

$$
\mathscr{L}_{2}=\left[\begin{array}{cccccccccccc}
d_{0}+(N-1) a & -a & -a & \cdots & -a & -a & -d_{0} & 0 & 0 & \cdots & 0 & 0 \\
0 & d+a_{0} & 0 & \cdots & 0 & -a_{0} & 0 & -d & 0 & \cdots & 0 & 0 \\
0 & -a_{0} & d+a_{0} & \cdots & 0 & 0 & 0 & 0 & -d & \cdots & 0 & 0 \\
\vdots & \vdots & \vdots & \ddots & \vdots & \vdots & \vdots & \vdots & \vdots & \ddots & \vdots & \vdots \\
0 & 0 & 0 & \cdots & d+a_{0} & 0 & 0 & 0 & 0 & \cdots & -d & 0 \\
0 & 0 & 0 & \cdots & -a_{0} & d+a_{0} & 0 & 0 & 0 & \cdots & 0 & -d \\
-d_{0} & 0 & 0 & \cdots & 0 & 0 & d_{0}+(N-1) a & -a & -a & \cdots & -a & -a \\
0 & -d & 0 & \cdots & 0 & 0 & 0 & d+a_{0} & 0 & \cdots & 0 & -a_{0} \\
0 & 0 & -d & \cdots & 0 & 0 & 0 & -a_{0} & d+a_{0} & \cdots & 0 & 0 \\
\vdots & \vdots & \vdots & \ddots & \vdots & \vdots & \vdots & \vdots & \vdots & \ddots & \vdots & \vdots \\
0 & 0 & 0 & \cdots & -d & 0 & 0 & 0 & 0 & \cdots & d+a_{0} & 0 \\
0 & 0 & 0 & \cdots & 0 & -d & 0 & 0 & 0 & \cdots & -a_{0} & d+a_{0}
\end{array}\right] .
$$

It can also be deduced that the characteristic polynomial of Network II is

$$
\begin{aligned}
& \left|\lambda I-\widetilde{\mathscr{L}}_{2}\right|=\left|\begin{array}{cccccc}
\lambda-(N-1) a & a & a & \cdots & a & a \\
0 & \lambda-a_{0} & 0 & \cdots & 0 & a_{0} \\
0 & a_{0} & \lambda-a_{0} & \cdots & 0 & 0 \\
\vdots & \vdots & \vdots & \ddots & \vdots & \vdots \\
0 & 0 & 0 & \cdots & \lambda-a_{0} & 0 \\
0 & 0 & 0 & \cdots & a_{0} & \lambda-a_{0}
\end{array}\right|_{N \times N} \\
& \left|\begin{array}{cccccc}
\lambda-2 d_{0}-(N-1) a & a & a & \cdots & a & a \\
0 & \lambda-2 d-a_{0} & 0 & \cdots & 0 & a_{0} \\
0 & a_{0} & \lambda-2 d-a_{0} & \cdots & 0 & 0 \\
\vdots & \vdots & \vdots & \ddots & \vdots & \vdots \\
0 & 0 & 0 & \cdots & \lambda-2 d-a_{0} & 0 \\
0 & 0 & 0 & \cdots & a_{0} & \lambda-2 d-a_{0}
\end{array}\right|_{N \times N} \\
& =[\lambda-(N-1) a]\left[(-1)^{N} a_{0}^{N-1}+\left(\lambda-a_{0}\right)^{N-1}\right]\left[\lambda-2 d_{0}-(N-1) a\right]\left[(-1)^{N} a_{0}^{N-1}+\left(\lambda-2 d-a_{0}\right)^{N-1}\right] .
\end{aligned}
$$


Therefore, when $N$ is odd, the eigenvalues $\lambda$ of $\mathscr{L}_{2}$ are $\underbrace{0, \ldots, 0}_{(N-1) / 2}, \underbrace{2 a_{0}, \ldots, 2 a_{0}}_{(N-1) / 2},(N-1) a, \underbrace{2 d+2 a_{0}, \ldots, 2 d+2 a_{0}}_{(N-1) / 2}$ $2 d, \ldots, 2 d, 2 d_{0}+(N-1) a$. When $N$ is even, the eigen$(N-1) / 2$

values $\lambda$ of $\mathscr{L}_{2}$ are $\underbrace{0, \ldots, 0}_{N-1}, \underbrace{2 d, \ldots, 2 d}_{N-1},(N-1) a$, $2 d_{0}+(N-1) a$.

The eigenvalues of the supra-Laplacian matrix $\mathscr{L}_{2}$ of Network II are different because of the odd and even number of subnetwork nodes. Here, we analyze the synchronizability of Network II according to the odd and even number of subnetwork nodes.

(1) $N$ is odd. $\lambda_{2}=\min \left\{2 a_{0}, 2 d\right\}, \quad R=\left(\lambda_{\max } / \lambda_{2}\right)=$ $\left(\left(2 d_{0}+(N-1) a\right) / \min \left\{2 a_{0}, 2 d\right\}\right)$. Variations of $\lambda_{2}, R$ of Network II are summarized in Table 5.

According to the MSF theory, when the synchronous region is unbounded, the synchronizability is determined by $\lambda_{2}=\min \left\{2 a_{0}, 2 d\right\}$. The larger $\lambda_{2}$ is, the stronger the synchronizability is. When the synchronous region is bounded, the synchronizability of Network II is determined by $R=\left(\left(2 d_{0}+(N-1) a\right) / \min \left\{2 a_{0}, 2 d\right\}\right)$. The smaller $R$ is, the stronger the synchronizability is. The relationship between the synchronizability and the structural parameters is shown in Table 6.

It can be seen from Table 6 that when the synchronous region is unbounded, the synchronizability is related to $a_{0}, d$, and with the increase of $a_{0}, d$, the synchronizability will strengthen. When the synchronous region is bounded, the synchronizability is related to $a_{0}$. When the intralayer coupling strength between the leaf node is small, the increase of $a_{0}$ enhances the synchronizability, while the increase of $N, a, d_{0}$ weakens the synchronizability. When the interlayer coupling strength between the leaf node is weak, the synchronizability strengthens with the increase of $d$ but weakens with the increase of $N, a, d_{0}$.

(2) $N$ is even. $\lambda_{2}=2 d, \lambda_{\max }=2 d_{0}+(N-1) a$, $R=\left(\lambda_{\max } / \lambda_{2}\right)=\left(\left(2 d_{0}+(N-1) a\right) / 2 d\right)$.

The variation of $\lambda_{2}, R$ of Network II is concluded in Table 7.

According to the MSF theory, when the network synchronous region is unbounded, the synchronizability is determined by $\lambda_{2}=2 d$. When the network synchronous region is bounded, the network synchronizability of Network II is determined by $R=\left(\left(2 d_{0}+(N-1) a\right) / 2 d\right)$. Whether the synchronous region is unbounded or bounded is independent of the intralayer coupling strength between leaf nodes $a_{0}$.

Therefore, the synchronizability is enhanced with the increase of $d$ in the unbounded synchronous region. The increase of $d$ enhances the synchronizability of Network II, but the increase of $N, a, d_{0}$ weakens the synchronizability in the bounded synchronous region.
4.3. Numerical Simulation of Network II Synchronizability. The section on the theoretical results shows that, in Network II, when the number of nodes in each layer of subnetwork is odd, the synchronizability is related to the number of subnetwork nodes, the intralayer coupling strength from the hub node to the leaf node, and the intralayer coupling strength between the leaf nodes, the interlayer coupling strength between the hub nodes, and the interlayer coupling strength between the leaf nodes. When the number of subnetwork nodes is even, the synchronizability is related to the number of subnetwork nodes, the intralayer coupling strength between the hub node and the leaf node, the interlayer coupling strength between the hub nodes, and the interlayer coupling strength between the leaf nodes. In the following numerical simulations, we will take $N=199$ (odd) and $N=200$ (even) separately to carry out numerical experiments to verify the above theoretical results.

(1) $N$ is odd. Take $a=1, a_{0}=0.5, d=1, d_{0}=0.1$ to examine the synchronizability of Network II with the change of the number of subnetwork nodes $N$ for $a_{0}>d$, and take $a=1, a_{0}=0.5, d=0.3, d_{0}=0.1$ to investigate the synchronizability as the number of subnetwork nodes $N$ changes for $a_{0}<d$, as shown in Figure 10. It can be seen from Figure 10 that for the case of the unbounded synchronous region (Figure 10(a)), when $a_{0}>d, \lambda_{2}$ is unchanged with the increase of $N$. When $a_{0}<d, \lambda_{2}$ remains unchanged with the increase of $N$; then, the synchronizability is invariant with the increase of $N$. For the case of the bounded synchronous region (Figure 10(b)), $R$ increases with the increase of $N$ in both $a_{0}>d$ and $a_{0}<d$, indicating that the greater the number of subnetwork nodes, the more unfavorable the synchronizability.

Take $N=199, a_{0}=0.5, d=1, d_{0}=0.1$ to investigate the synchronizability with the change of the intralayer coupling strength $a$ from the hub nodes to the leaf nodes, and take $N=199, a_{0}=0.5, d=0.3, d_{0}=0.1$ to investigate the change in synchronizability with the intralayer coupling strength $a$ from the hub nodes to the leaf nodes, as shown in Figure 11. Figure 11(a) shows that neither $a_{0}>d$ nor $a_{0}<d, \lambda_{2}$ is not affected by $a$, indicating that $a$ has no influence on the synchronizability, in the unbounded synchronous region. Figure 11(b) displays that when the synchronous region is bounded, $R$ increases in both $a_{0}>d$ and $a_{0}<d$ with the increase of $a$. Moreover, the interlayer coupling strength between the leaf nodes $d$ has a great influence on the synchronizability, which makes the synchronizability weaken faster. The synchronizability weakens with the increase of $a$, and the larger $a$ is, the more unfavorable the synchronizability is.

Notice from Figure $12(N=199, a=1, d=1$, $d_{0}=0.1$ ) how the synchronizability changes with the intralayer coupling strength $a_{0}$ between the leaf 
TABLE 5: The change of $\lambda_{2}$ and $R$ with the increase of $N, a, a_{0}, d$, and $d_{0}$ of Network II when $N$ is odd.

\begin{tabular}{|c|c|c|c|c|c|c|}
\hline & & Increase of $N$ & Increase of $a$ & Increase of $a_{0}$ & Increase of $d$ & Increase of $d_{0}$ \\
\hline$\lambda_{2}=\min \left\{2 d, 2 a_{0}\right\}$ & $\begin{array}{l}d>a_{0} \\
d<a_{0}\end{array}$ & $\begin{array}{l}\text { Unchanged } \\
\text { Unchanged }\end{array}$ & $\begin{array}{l}\text { Unchanged } \\
\text { Unchanged }\end{array}$ & $\begin{array}{l}\text { Increased } \\
\text { Unchanged }\end{array}$ & $\begin{array}{l}\text { Unchanged } \\
\text { Increased }\end{array}$ & $\begin{array}{l}\text { Unchanged } \\
\text { Unchanged }\end{array}$ \\
\hline$R=\left(\left(2 d_{0}+(N-1) a\right) / \min \left\{2 d, 2 a_{0}\right\}\right)$ & $\begin{array}{l}d>a_{0} \\
d<a_{0}\end{array}$ & $\begin{array}{l}\text { Increased } \\
\text { Increased }\end{array}$ & $\begin{array}{l}\text { Increased } \\
\text { Increased }\end{array}$ & $\begin{array}{l}\text { Decreased } \\
\text { Unchanged }\end{array}$ & $\begin{array}{l}\text { Unchanged } \\
\text { Decreased }\end{array}$ & $\begin{array}{l}\text { Increased } \\
\text { Increased }\end{array}$ \\
\hline
\end{tabular}

TABle 6: The change of synchronizability with the increase of $N, a, a_{0}, d$, and $d_{0}$ of Network II when $N$ is odd.

\begin{tabular}{|c|c|c|c|c|c|c|}
\hline & & Increase of $N$ & Increase of $a$ & Increase of $a_{0}$ & Increase of $d$ & Increase of $d_{0}$ \\
\hline $\begin{array}{l}\text { The unbounded synchronous } \\
\text { region } \\
\text { The bounded synchronous } \\
\text { region }\end{array}$ & $\begin{array}{c}\lambda_{2}=2 a_{0} \\
\lambda_{2}=2 d \\
R=\left(\left(2 d_{0}+(N-1) a\right) / 2 a_{0}\right) \\
R=\left(\left(2 d_{0}+(N-1) a\right) / 2 d\right)\end{array}$ & $\begin{array}{l}\text { Unchanged } \\
\text { Unchanged } \\
\text { Weakened } \\
\text { Weakened }\end{array}$ & $\begin{array}{l}\text { Unchanged } \\
\text { Unchanged } \\
\text { Weakened } \\
\text { Weakened }\end{array}$ & $\begin{array}{l}\text { Strengthened } \\
\text { Unchanged } \\
\text { Strengthened } \\
\text { Unchanged }\end{array}$ & $\begin{array}{l}\text { Unchanged } \\
\text { Strengthened } \\
\text { Unchanged } \\
\text { Strengthened }\end{array}$ & $\begin{array}{l}\text { Unchanged } \\
\text { Unchanged } \\
\text { Weakened } \\
\text { Weakened }\end{array}$ \\
\hline
\end{tabular}

TABLE 7: The change of $\lambda_{2}$ and $R$ with $N, a, d$, and $d_{0}$ of Network II when $N$ is even.

\begin{tabular}{|c|c|c|c|c|}
\hline & Increase of $N$ & Increase of $a$ & Increase of $d$ & Increase of $d_{0}$ \\
\hline $\begin{array}{l}\lambda_{2}=2 d \\
R=\left(\left(2 d_{0}+(N-1) a\right) / 2 d\right)\end{array}$ & $\begin{array}{l}\text { Unchanged } \\
\text { Increase }\end{array}$ & $\begin{array}{l}\text { Unchanged } \\
\text { Increased }\end{array}$ & $\begin{array}{l}\text { Increased } \\
\text { Decreased }\end{array}$ & $\begin{array}{l}\text { Unchanged } \\
\text { Increased }\end{array}$ \\
\hline
\end{tabular}

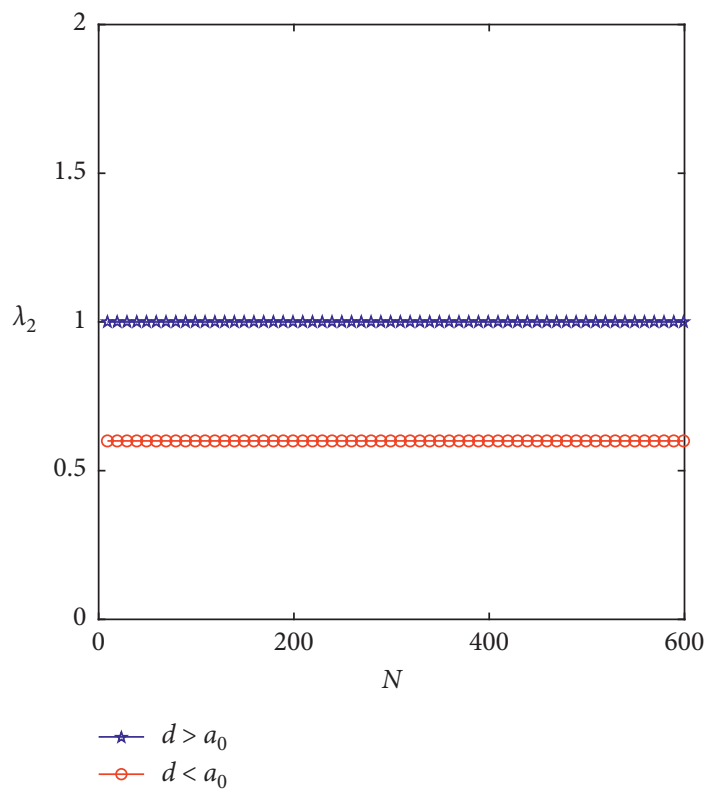

(a)

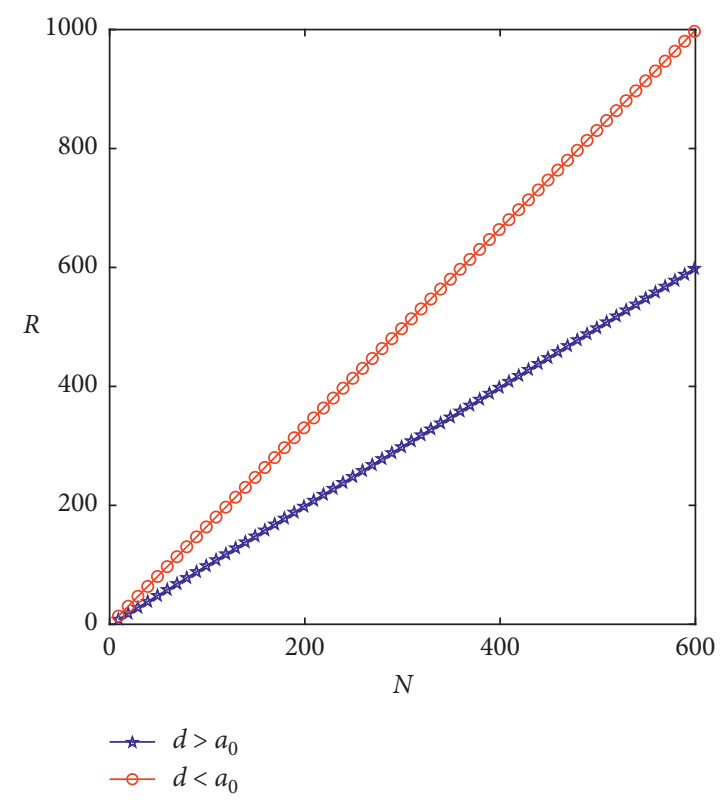

(b)

FIgURE 10: When $N$ is odd, the synchronizability of Network II changes with the number of the subnetwork nodes $N$. (a) $\lambda_{2}$ changes with $N$ ( $N$ changes from 9 to 599); (b) $R$ changes with $N$ ( $N$ changes from 9 to 599).

nodes. For the case of the unbounded synchronous region (Figure 12(a)), when $a_{0}=d, \lambda_{2}$ increases with the increase of $a_{0}$, and when $a_{0}$ increases to the threshold value $d, \lambda_{2}$ remains unchanged with the increase of $a_{0}$. In this case, $d$ is the optimal structural parameter that enables Network II to reach the optimal synchronizability. The synchronizability at first strengthens and then remains unchanged with the increase of $a_{0}$. For the case of the bounded synchronous region (Figure 12(b)), when $a_{0}=d, R$ rapidly decreases with the increase of $a_{0}$; when $a_{0}$ increases to the threshold value $d, R$ starting along with the increase of $a_{0}$ remains invariant; thus, the threshold value $d$ is to achieve the optimal synchronizability when the structure parameters are optimal. Therefore, with the increase of $a_{0}$, the synchronizability strengthens before remaining invariant. 


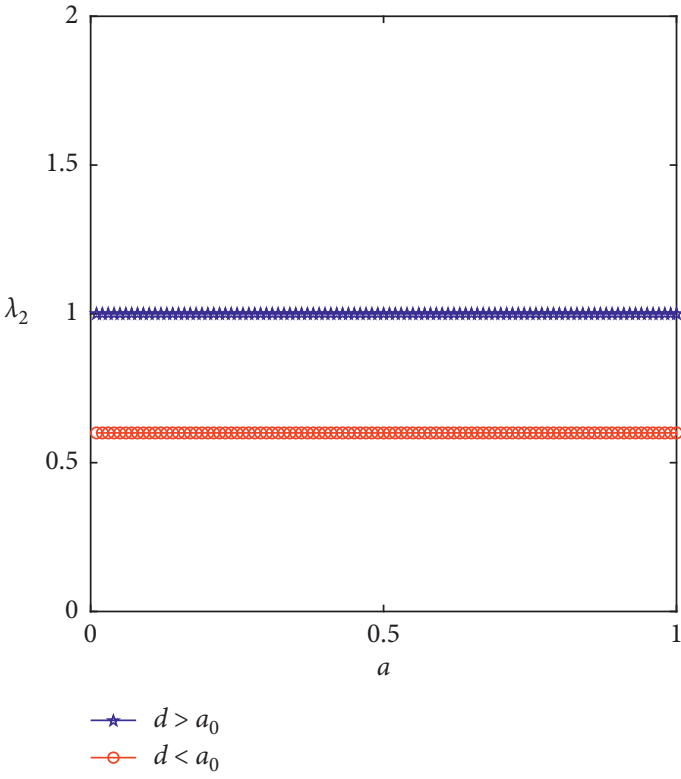

(a)

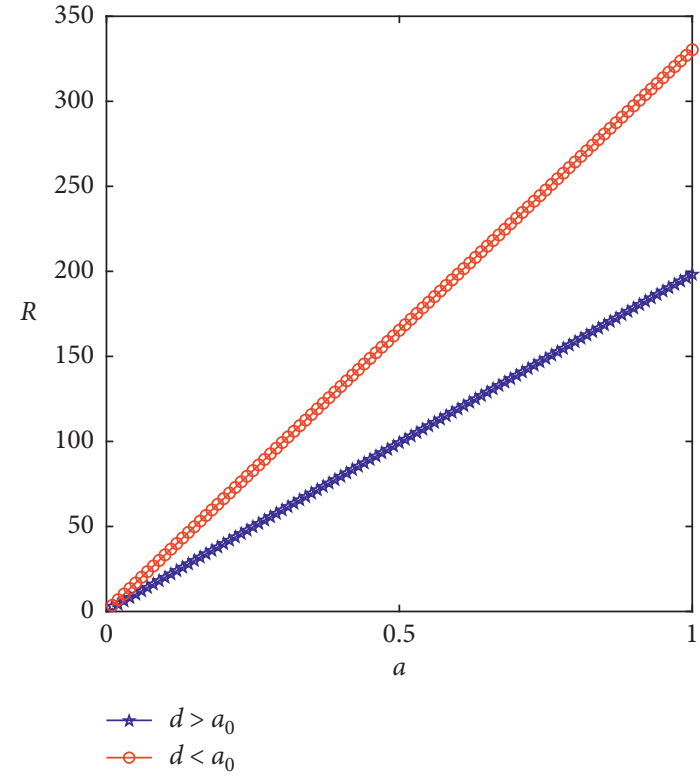

(b)

Figure 11: When $N$ is odd, the synchronizability of Network II changes with the intralayer coupling strength $a$ from the leaf nodes to the hub nodes. (a) $\lambda_{2}$ changes with $a$ ( $a$ changes from 0.01 to 1 ); (b) $R$ changes with $a$ ( $a$ changes from 0.01 to 1 ).

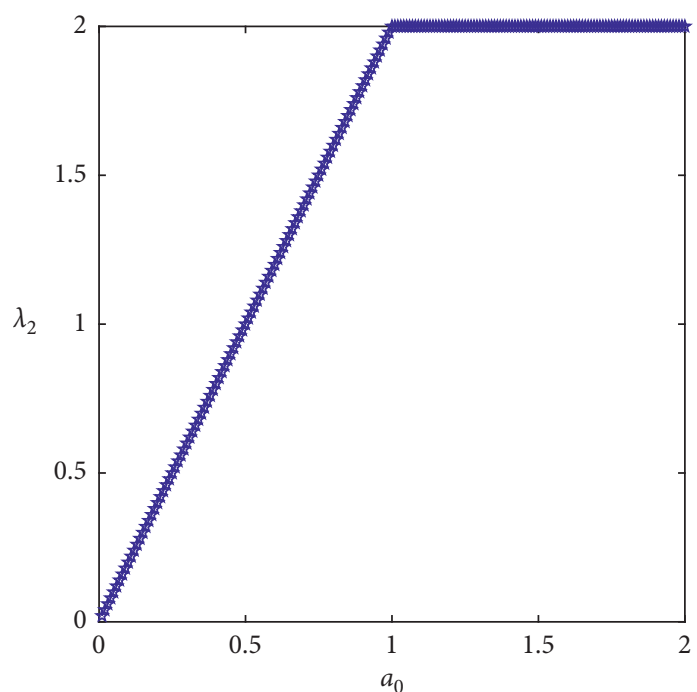

(a)

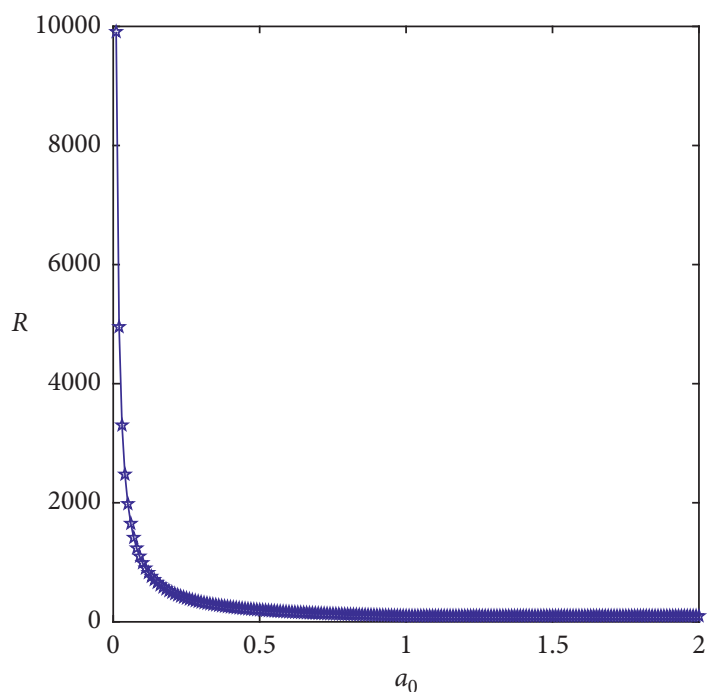

(b)

Figure 12: When $N$ is odd, the synchronizability of Network II changes with the intralayer coupling strength $a_{0}$ of the leaf nodes. (a) $\lambda_{2}$ changes with $a_{0}$ ( $a_{0}$ changes from 0.01 to 2 ); (b) $R$ changes with $a_{0}$ ( $a_{0}$ changes from 0.01 to 2 ).

As shown in Figure 13, take $N=199, a=1$, $a_{0}=0.5, d_{0}=0.1$ to examine the synchronizability with the change of the interlayer coupling strength $d$ between the leaf nodes. For the case of the unbounded synchronous region (Figure 13(a)), when $a_{0}>d, \lambda_{2}$ increases with the increase of $d$. When $d$ increases to the threshold value $a_{0}, \lambda_{2}$ remains unchanged with the increase of $d$; at this time, $d$ is the optimal structural parameter to reach the optimal synchronizability. It shows that the synchronizability strengthens at first and then remains unchanged with the increase of $d$. For the case of the bounded synchronous region (Figure 13(b)), when $a_{0}>d, R$ decreases rapidly with the increase of $d$. When $d$ increases to the threshold value $a_{0}, R$ does not change with the increase of $d$. The threshold value $a_{0}$ is the optimal structural parameter for optimal synchronizability. It is concluded that, with the increase of $d$, the synchronizability will strengthen at first and then remains unchanged.

The simulation results are shown in Figure 14. Take $N=199, a=1, a_{0}=0.5, d=1$ to investigate how the synchronizability changes with the interlayer 


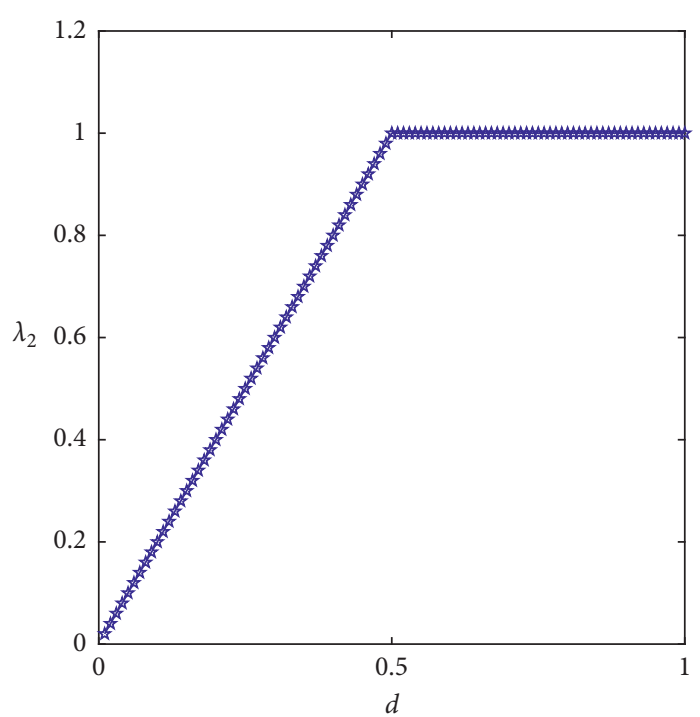

(a)

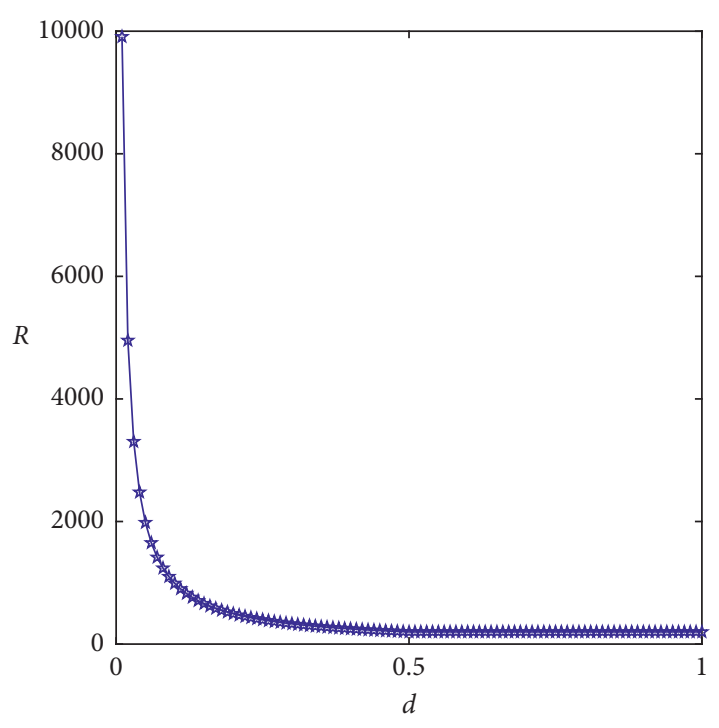

(b)

FIGURE 13: When $N$ is odd, the synchronizability of Network II changes with the interlayer coupling strength $d$ of the leaf nodes. (a) $\lambda_{2}$ changes with $d$ ( $d$ changes from 0.01 to 1 ); (b) $R$ changes with $d$ ( $d$ changes from 0.01 to 1 ).

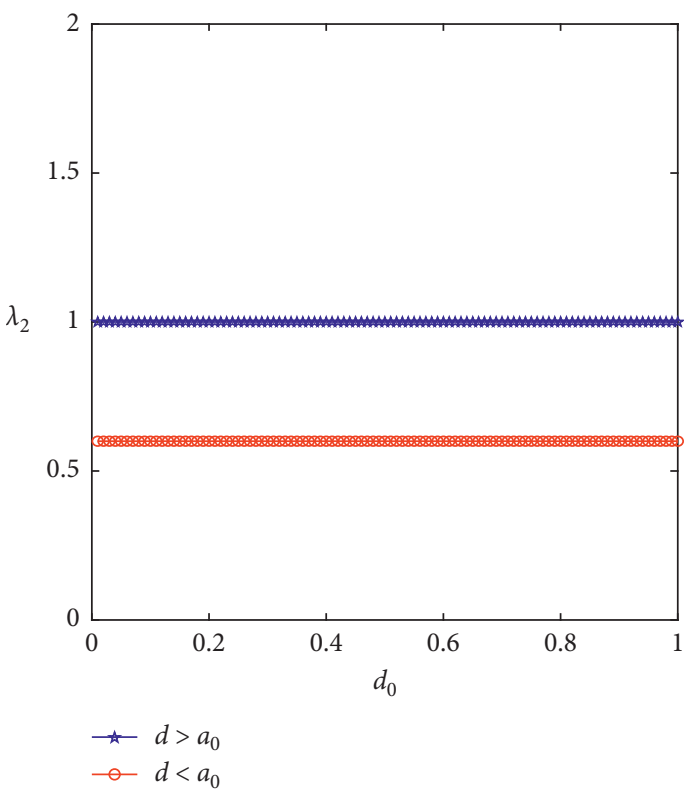

(a)

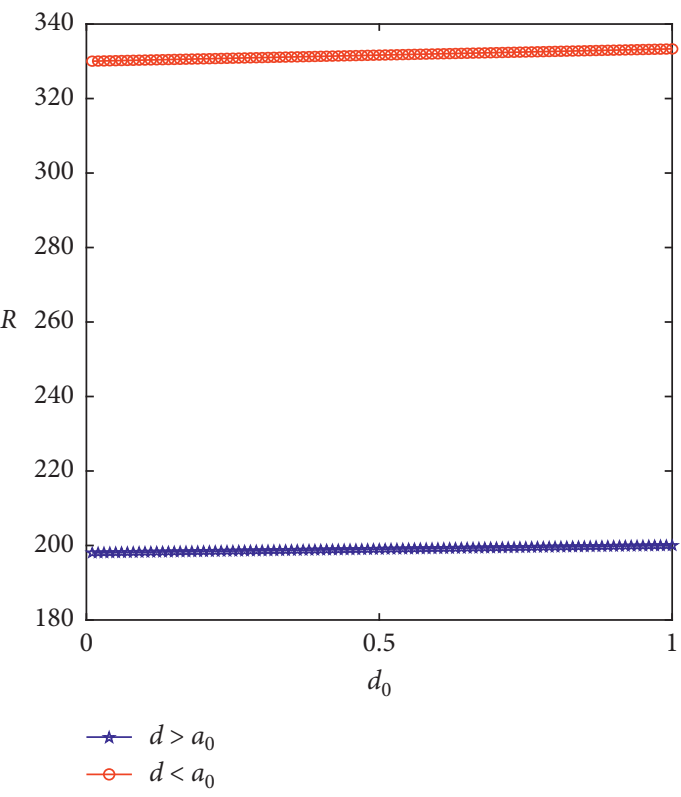

(b)

Figure 14: When $N$ is odd, the synchronizability of Network II varies with the interlayer coupling strength $d_{0}$ of the leaf nodes. (a) $\lambda_{2}$ changes with $d_{0}$ ( $d_{0}$ changes from 0.01 to 1 ); (b) $R$ changes with $d_{0}$ ( $d_{0}$ changes from 0.01 to 1 ).

coupling strength $d_{0}$ (when $a_{0}>d$ ) between the hub nodes. Take $N=199, a=1, a_{0}=0.5, d=0.3$ to investigate how the synchronizability changes with the interlayer coupling strength $d_{0}$ (when $a_{0}<d$ ) between the hub nodes. In the unbounded synchronous region (Figure 14(a)), either $a_{0}>d$ or $a_{0}<d$, and $\lambda_{2}$ are basically unchanged with the increase of $d_{0}$, indicating that the synchronizability is unchanged with the increase of $d_{0}$. In the bounded synchronous region (Figure 14(b)), $R$ increases slowly with the increase of $d_{0}$ in both $a_{0}>d$ and $a_{0}<d$. Therefore, the synchronizability weakens with the increase of $d_{0}$.

(2) $N$ is even. For Network II with the even of the subnetwork nodes, it is found that the synchronizability is influenced by the number of subnetwork nodes, the intralayer coupling strength from the hub node to the leaf node, the interlayer 


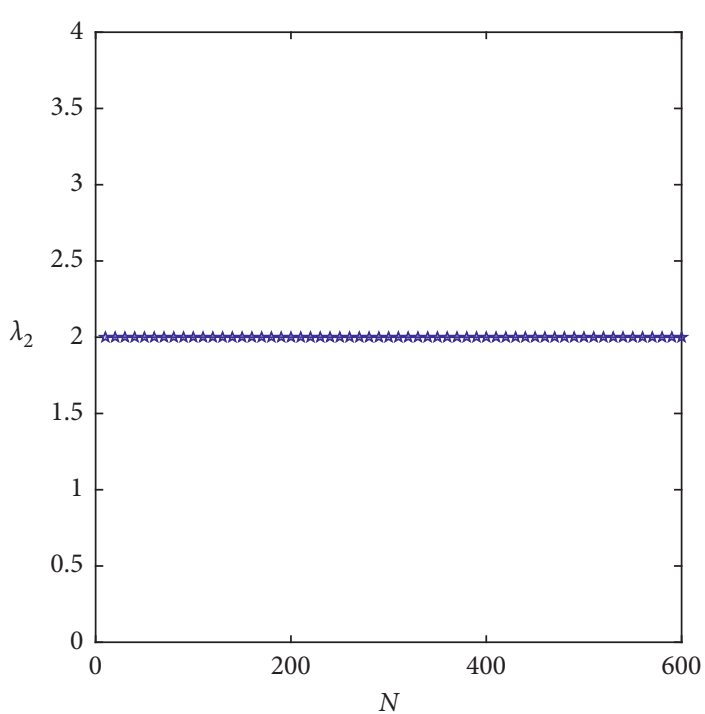

(a)

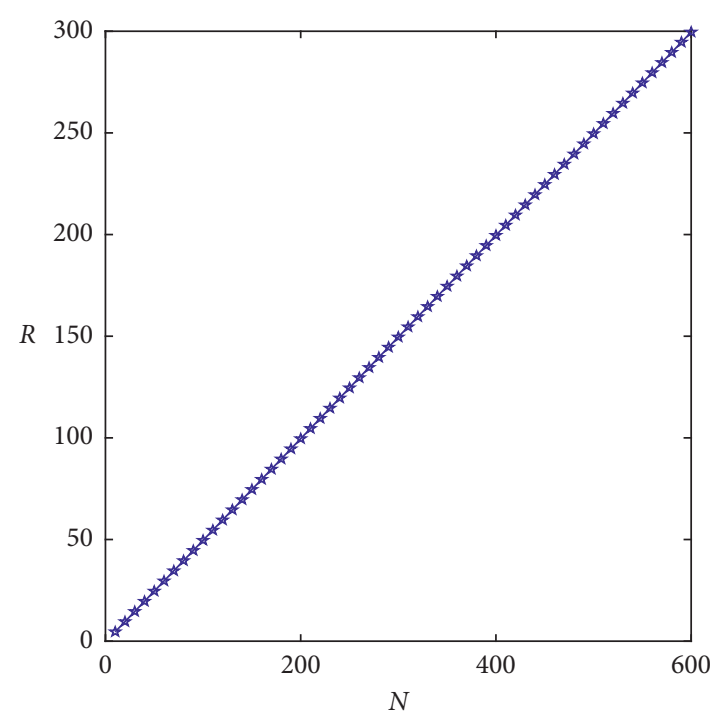

(b)

FIGURE 15: When $N$ is even, the synchronizability of Network II changes with the number of subnetwork nodes $N$. (a) $\lambda_{2}$ changes with $N$ ( $N$ changes from 10 to 600 ); (b) $R$ changes with $N$ ( $N$ changes from 10 to 600 ).

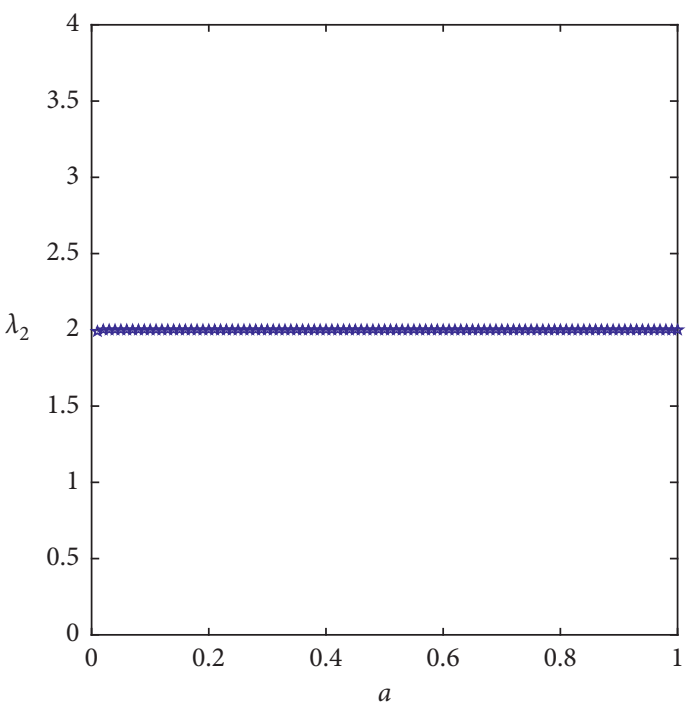

(a)

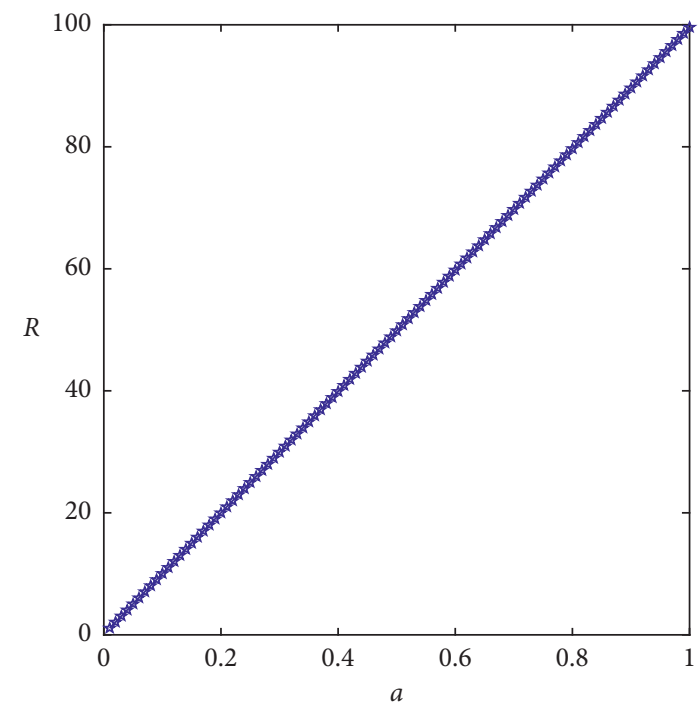

(b)

FIGURE 16: When $N$ is even, the synchronizability of Network II changes with the intralayer coupling strength $a$ from the leaf nodes to the hub nodes. (a) $\lambda_{2}$ changes with $a$ ( $a$ changes from 0.01 to 1 ); (b) $R$ changes with $a$ ( $a$ changes from 0.01 to 1 ).

coupling strength between the leaf nodes, and the interlayer coupling strength between the hub nodes, which are the same as those when the number of the subnetwork nodes is odd for $a_{0}>d$, namely, Figure 10 (the red line) and Figure 15, Figure 11 (the red line) and Figure 16, Figure 13 (when $d<a_{0}$ ) and Figure 17, and Figure 14 (the red line) and Figure 18. The synchronizability strengthens with the increase of $d$ in the unbounded synchronous region. The synchronizability is enhanced with the increase of $d$ but weakens with the increase of $N, a, d_{0}$, in the bounded synchronous region. Moreover, the intralayer coupling strength $a_{0}$ between the leaf nodes has no effect on the synchronizability when $N$ is even. At this time, the edges between the intralayer leaf nodes are removed, which is the twolayer unidirectional coupled star network from the hub nodes to the leaf node. The above conclusions are the same as the simulation results of the star network with unidirectional coupling between the three-layer hub nodes and the leaf node in [30]. 


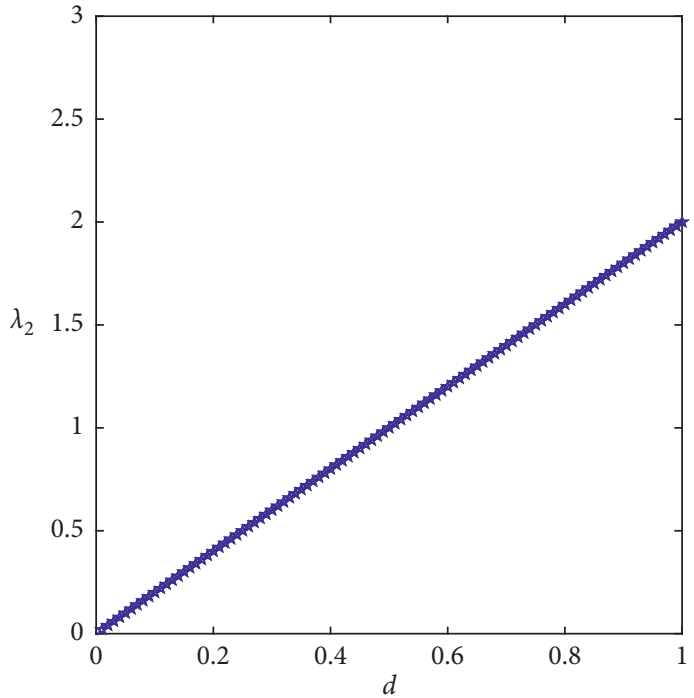

(a)

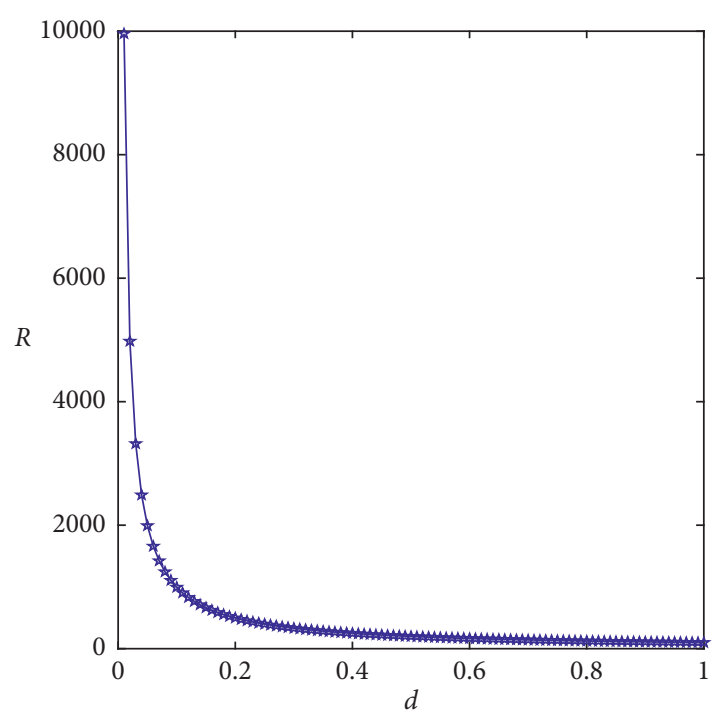

(b)

Figure 17: When $N$ is even, the synchronizability of Network II changes with the interlayer coupling strength $d$ between the leaf nodes. (a) $\lambda_{2}$ changes with $d$ ( $d$ changes from 0.01 to 1 ); (b) $R$ changes with $d$ ( $d$ changes from 0.01 to 1 ).

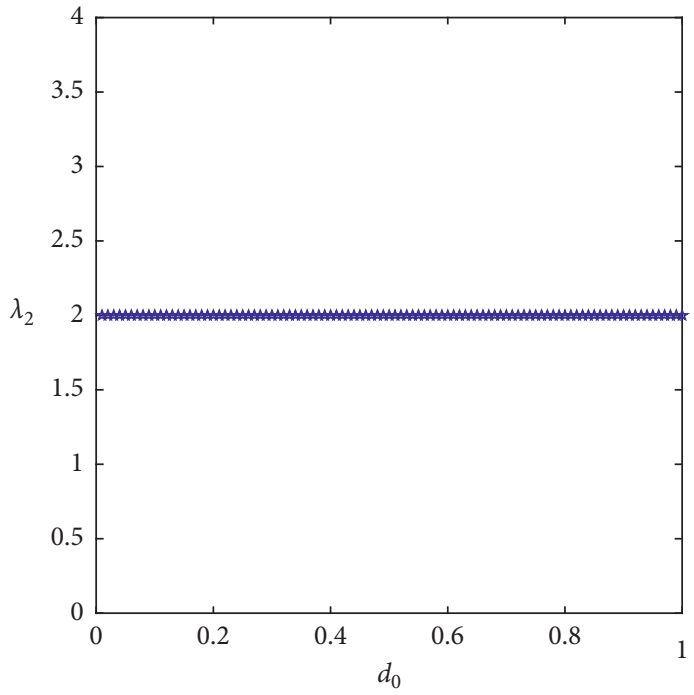

(a)

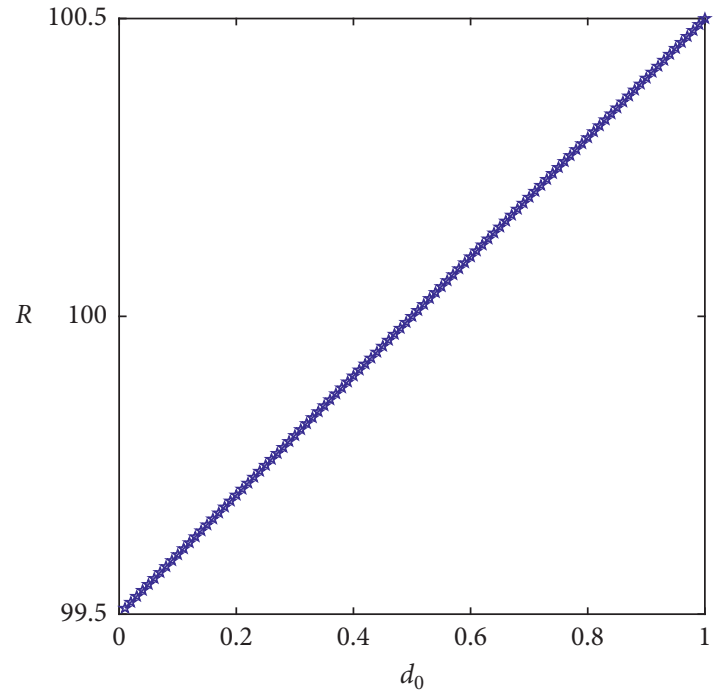

(b)

FIgURE 18: When $N$ is even, the synchronizability of Network II varies with the interlayer coupling strength $d_{0}$ of the leaf nodes. (a) $\lambda_{2}$ changes with $d_{0}$ ( $d_{0}$ changes from 0.01 to 1 ); (b) $R$ changes with $d_{0}$ ( $d_{0}$ changes from 0.01 to 1 ).

\section{Conclusion and Discussion}

In this article, we study the eigenvalue spectrum and synchronizability of two types of double-layer hybrid directional coupling star-ring network. One is the double-layer hybrid directional coupled star-ring networks from the leaf nodes to the hub nodes (Network I); the other is the doublelayer hybrid directional coupling star-ring network (Network II) from the hub node to the leaf node. The eigenvalue spectrum of the two types of double-layer star-ring networks is derived strictly in theory, and the relationship between the synchronization and the structural parameters is analyzed. Different from the results of other similar networks, the parity of the number of star-ring subnetwork nodes in the two kinds of networks studied in this article has a certain impact on the synchronization, and the synchronization with odd or even of the subnetwork nodes has a different dependence on the structure parameters. Deng et al. [32] studied multilayer undirected star-ring and star networks. By comparing the simulation results of the interlayer coupling strength in this article with the case that the interlayer coupling strength is not equal in [32], it is found that the 
influence of the interlayer coupling strength on the network synchronization ability is the same only in the case of Network I and the odd number of subnetwork nodes. The rest of the cases are that the influence of synchronizability is the same between the interlayer coupling strength of the leaf node in this article and multilayer undirected star and starring networks of the interlayer coupling strength of the hub node, and the interlayer coupling strength between hub nodes in our article has the same effect on the synchronization as the interlayer coupling strength between leaf nodes of the multilayer undirected star and star-ring networks.

For Network I, in the case that the synchronous region is unbounded, no matter whether the number of subnetwork nodes is odd or even, the synchronizability is enhanced with the increase of the intralayer coupling strength from the leaf nodes to the hub nodes and the interlayer coupling strength between the hub nodes. In the case that the synchronous region is bounded, when the intralayer coupling strength from the leaf nodes to the hub nodes is weaker, the synchronizability strengthens with the increase of the intralayer coupling strength from the leaf nodes to the hub nodes but weakens with the increase of the interlayer coupling strength between the leaf nodes. When the interlayer coupling strength between the hub nodes is weaker, without the parity of the number of subnetwork nodes, the synchronizability is enhanced with the increase of the interlayer coupling strength between the hub nodes but weakens with the increase of the intralayer coupling strength between the leaf nodes to the hub nodes and the interlayer coupling strength between the leaf nodes. When the number of subnetwork nodes is odd, the synchronizability weakens with the increase of the intralayer coupling strength between the leaf nodes. When the number of subnetwork nodes is even, the intralayer coupling strength between the leaf nodes has no effect on network synchronizability; in this case, studying network synchronizability can remove the edge between the leaf nodes of the ring, to reduce the physical memory and to improve the work efficiency, and the results and three layers of the leaf nodes pointing to the hub nodes of unidirectionally coupling conclusion are consistent with the star networks in [30].

For Network II, the intralayer coupling strength between the leaf nodes has an influence on the synchronizability when the number of subnetwork nodes is odd but not when the number of subnetwork nodes is even. When the number of subnetwork nodes is even, the conclusion is the same as that of the three-layer star networks, unidirectionally coupling from the hub nodes to the leaf nodes in [30]. Therefore, when the number of subnetwork nodes is even, synchronizability research can reduce physical memory and improve the working efficiency by deleting the edges between the leaf nodes in the ring. In the case that the synchronous region is unbounded, when the number of subnetwork nodes is odd, the synchronizability is enhanced with the increase of the intralayer coupling strength between the leaf nodes and the interlayer coupling strength between the leaf nodes. When the number of nodes in the subnetwork is even, the synchronizability is enhanced with the increase of the interlayer coupling strength between the leaf nodes. For the bounded synchronous region, when the intralayer coupling strength between the leaf nodes is weaker, in the case that the number of subnetwork nodes is odd, the synchronizability is enhanced with the increase of the intralayer coupling strengthened between the leaf nodes, but with the increase of the number of subnetwork nodes, the intralayer coupling strength between the hub nodes to the leaf nodes and the interlayer coupling strength between the hub nodes are weakened. When the interlayer coupling strength between the leaf nodes is weaker, whether the number of nodes in the subnetwork is odd or even, the synchronizability strengthens with the increase of the interlayer coupling strength between the leaf nodes but weakens with the increase of the number of nodes in the subnetwork, the intralayer coupling strength between the hub nodes to the leaf nodes, and the interlayer coupling strength between the hub nodes.

\section{Prospect}

Due to the large scale and complex structure of multilayer networks, its network dynamics properties are also very abundant, but it is very difficult to research, especially to obtain the results of the theoretical derivation. The theoretical research of the network in this article can be used to study the dynamics of more general multilayer hybrid directional networks. The simulation results can help better to understand the design and control of real complex systems. Here, we obtain the theoretical results of the eigenvalue spectrum of two kinds of two-layer hybrid directionally coupled star-ring networks. However, if we generalize the model to any multilayer network, it will be more challenging to solve its eigenvalue spectrum. Secondly, what is the eigenvalue spectrum and synchronizability of the two-layer network model that we studied, when there is a direction between the hub nodes of the interlayer or between the leaf nodes of the interlayer? If the intralayer coupling strength between the leaf nodes is unchanged, how do other parameters change so that the synchronizability remains unchanged? We will continue to devote ourselves to the in-depth study of these issues. There are various multilayer directional networks in the real world. At present, the theoretical model of multilayer directional networks is still weak in practical application, mainly because of the gap between the theoretical model and the actual data. With the further investigation of the theory of multilayer hybrid directional network, the theoretical model will be more and more close to the real system, and the practical application will be increasingly common.

\section{Data Availability}

The MATLAB running codes of the numerical simulation data in this paper are included in the supplementary file.

\section{Conflicts of Interest}

The authors declare that they have no conflicts of interest. 


\section{Acknowledgments}

This project was supported by the Natural Science Foundation of Guangxi (no. 2018GXNSFAA138095) and the National Natural Science Foundation of China (no. 61563013).

\section{Supplementary Materials}

The procedures are MATLAB language code, and the data are obtained by running codes provided. (Supplementary Materials)

\section{References}

[1] P. H. Feng, Z. W. Gong, and D. Y. Sun, "Research on interpersonal relationship network of pyramid scheme organization based on social network: a case study," International Journal of Modern Physics C, vol. 30, no. 2, p. 15, 2019.

[2] S. Sinha, "Complexity vs. stability in small-world net-works," Physica A: Statistical Mechanics and Its Applications, vol. 346, no. 1, pp. 147-153, 2004.

[3] M. E. J. Newman, "The structure and function of complex networks," SIAM Review, vol. 45, no. 2, pp. 167-256, 2003.

[4] D. J. Watts and S. H. Strogatz, "Collective dynamics of "smallworld” networks," Nature, vol. 393, no. 2, pp. 440-442, 1998.

[5] F. Mori and T. Odagaki, "The Laplacian spectra of smallworld networks," Journal of the Physical Society of Japan, vol. 73, no. 12, pp. 3294-3298, 2004.

[6] Y. Wu, Y. Shang, M. Chen, C. Zhou, and J. Kurths, "Synchronization in small-world networks," Chaos (Woodbury, N.Y.), vol. 18, no. 3, Article ID 037111, 2008.

[7] X. F. Wang and G. Chen, "Synchronization in scale-free dynamical networks: robustness and fragility," IEEE Transactions on Circuits and Systems I: Fundamental Theory and Applications, vol. 49, no. 1, pp. 54-62, 2002.

[8] J. Wu and L. Jiao, "Synchronization in complex dynamical networks with nonsymmetric coupling," Physica D: Nonlinear Phenomena, vol. 237, no. 19, pp. 2487-2498, 2008.

[9] H. Kang, K. Liu, and X. Fu, "Dynamics of an epidemic model with quarantine on scale-free networks," Physics Letters A, vol. 381, no. 47, pp. 3945-3951, 2017.

[10] B. Vaseghi, S. S. Hashemi, S. Mobayen, and A. Fekih, "Finite time chaos synchronization in time-delay channel and its application to satellite image encryption in OFDM communication systems," IEEE Access, vol. 9, pp. 21332-21344, 2021.

[11] B. Vaseghi, S. Mobayen, S. S. Hashemi, and A. Fekih, "Fast reaching finite time synchronization approach for chaotic systems with application in medical image encryption," IEEE Access, vol. 9, pp. 25911-25925, 2021.

[12] J. Mostafaee, S. Mobayen, B. Vaseghi et al., "Complex dynamical behaviors of a novel exponential hyper- chaotic system and its application in fast synchronization and color image encryption," Science Progress, vol. 104, no. 1, 2021.

[13] O. Mofid, M. Momeni, S. Mobayen, and A. Fekih, "A disturbance-observer-based sliding mode control for the robust synchronization of uncertain delayed chaotic systems: application to data security," IEEE Access, vol. 9, pp. 16546$16555,2021$.

[14] J. Mostafaee, S. Mobayen, B. Vaseghi et al., "Dynamical analysis and finite-time fast synchronization of a novel autonomous hyper-chaotic system," Journal of Intelligent Procedures in Electrical Technology, vol. 12, no. 47, 2021.
[15] H. Hong, M. Y. Choi, and B. J. Kim, "Synchronization on small-world networks," Physical Review. E, Statistical, Nonlinear, and Soft Matter Physics, vol. 65, no. 2, Article ID 026139, 2002.

[16] Y. Zhang and R. Xiao, "Synchronization of Kuramoto oscillators in small-world networks," Physica A: Statistical Mechanics and Its Applications, vol. 416, pp. 33-40, 2014.

[17] W. Zou and M. Zhan, "Complete synchronization in coupled limit-cycle systems,” EPL (Europhysics Letters), vol. 81, no. 1, Article ID 10006, 2008.

[18] Y. Q. Gu, C. Shao, and X. C. Fu, "Complete synchronization and stability of star-shaped complex networks," Chaos, Solitons and Fractals, vol. 28, no. 2, pp. 480-488, 2005.

[19] K. Li, S. Guan, X. Gong, and C.-H. Lai, "Synchronization stability of general complex dynamical networks with timevarying delays," Physics Letters A, vol. 372, no. 48, pp. 7133-7139, 2008.

[20] A. Arenas, A. Díaz-Guilera, J. Kurths, Y. Moreno, and C. Zhou, "Synchronization in complex networks," Physics Reports, vol. 469, no. 3, pp. 93-153, 2008.

[21] P. Kwangho, L. Ying-Cheng, G. Saurabh et al., "Synchronization in complex networks with a modular structure," Chaos (Woodbury, N.Y.), vol. 16, no. 1, Article ID 015105, 2006.

[22] Q. Y. Wang, G. R. Chen, Q. S. Lu et al., "Novel criteria of synchronization stability in complex networks with coupling delays," Physica A: Statistical Mechanics and Its Applications, vol. 378 , no. 2, pp. 527-536, 2006.

[23] M. E. Adilson, C. S. Zhou, and K. Jürgen, "Network synchronization, diffusion, and the paradox of heterogeneity," Physical Review. E, Statistical, Nonlinear, and Soft Matter Physics, vol. 71, no. 1, Article ID 016116, 2005.

[24] Z. Jia and G. M. Deng, "Mathematical model and cluster synchronization for a complex dynamical network with two types of chaotic oscillators," Journal of Applied Mathematics, vol. 2012, Article ID 595360, 12 pages, 2012.

[25] Y. L. Xi, Z. Y. Wu, and X. C. Fu, "Dynamical synchronization and stability of complex networks with multi-layer centers," Chaos, Solitons and Fractals, vol. 40, no. 2, pp. 635-641, 2007.

[26] J. Aguirre, R. Sevilla-Escoboza, R. Gutiérrez, D. Papo, and J. M. Buldú, "Synchronization of interconnected networks: the role of connector nodes," Physical Review Letters, vol. 112, no. 24, Article ID 248701, 2014.

[27] A. Solé-Ribalta, M. De Domenico, N. E. Kouvaris et al., "Spectral properties of the Laplacian of multiplex networks," Physical Review. E, Statistical, Nonlinear, and Soft Matter Physics, vol. 88, no. 3, Article ID 032807, 2013.

[28] M. M. Xu, J. A. Lu, and J. Zhou, "Synchronizability and eigenvalues of two-layer star networks," Acta Physica Sinica, vol. 65, no. 2, in Chinese, Article ID 028902, 2016.

[29] S. Gómez, A. Díaz-Guilera, J. Gómez-Gardeñes, C. J. PérezVicente, Y. Moreno, and A. Arenas, "Diffusion dynamics on multiplex networks," Physical Review Letters, vol. 110, no. 2, Article ID 028701, 2013.

[30] J. Sun, X.-X. Li, J.-H. Zhang, Y.-Z. Shen, and Y.-Y. Li, "Synchronizability and eigenvalues of multilayer star networks through unidirectionally coupling," Acta Physica Sinica, vol. 66, no. 18, in Chinese, Article ID 188901, 2017.

[31] Y. Deng, Z. Jia, G. M. Deng et al., "Eigenvalue spectrum and synchronizability of multiplex chain networks," Physica A: Statistical Mechanics and Its Applications, vol. 537, Article ID 122631, 2019.

[32] Y. Deng, Z. Jia, F. M. Yang et al., "Synchronizability of multilayer star and star-ring networks," Discrete Dynamics in 
Nature and Society, vol. 2020, Article ID 9143917, 20 pages, 2020.

[33] L. K. Tang, J. A. Lu, X. Q. Wu et al., "Impact of node dynamics parameters on topology identification of complex dynamical networks," Nonlinear Dynamics, vol. 73, no. 1-2, pp. 1081-1097, 2013.

[34] C. Li and G. Chen, "Synchronization in general complex dynamical networks with coupling delays," Physica A: Statistical Mechanics and Its Applications, vol. 343, pp. 263-278, 2004.

[35] L. M. Pecora and T. L. Carroll, "Master stability functions for synchronized coupled systems," International Journal of Bifurcation and Chaos, vol. 9, no. 12, pp. 2315-2320, 1999. 